\title{
ON THE RELATIONSHIP BETWEEN PHOTOSPHERIC FOOTPOINT MOTIONS AND CORONAL HEATING IN SOLAR ACTIVE REGIONS
}

\author{
A. A. van Ballegooijen ${ }^{1}$, M. Asgari-Targhi ${ }^{1}$, And M. A. Berger ${ }^{2}$ \\ ${ }^{1}$ Harvard-Smithsonian Center for Astrophysics, 60 Garden Street MS-15, Cambridge, MA 02138, USA \\ ${ }^{2}$ SECAM, University of Exeter, EX4 4QF, UK \\ Received 2014 January 13; accepted 2014 April 10; published 2014 May 6
}

\begin{abstract}
Coronal heating theories can be classified as either direct current (DC) or alternating current (AC) mechanisms, depending on whether the coronal magnetic field responds quasi-statically or dynamically to the photospheric footpoint motions. In this paper we investigate whether photospheric footpoint motions with velocities of $1-2 \mathrm{~km} \mathrm{~s}^{-1}$ can heat the corona in active regions, and whether the corona responds quasi-statically or dynamically to such motions (DC versus AC heating). We construct three-dimensional magnetohydrodynamic models for the Alfvén waves and quasi-static perturbations generated within a coronal loop. We find that in models where the effects of the lower atmosphere are neglected, the corona responds quasi-statically to the footpoint motions (DC heating), but the energy flux into the corona is too low compared to observational requirements. In more realistic models that include the lower atmosphere, the corona responds more dynamically to the footpoint motions (AC heating) and the predicted heating rates due to Alfvén wave turbulence are sufficient to explain the observed hot loops. The higher heating rates are due to the amplification of Alfvén waves in the lower atmosphere. We conclude that magnetic braiding is a highly dynamic process.
\end{abstract}

Key words: magnetohydrodynamics (MHD) - Sun: corona - Sun: magnetic fields - turbulence

Online-only material: animations, color figures

\section{INTRODUCTION}

It was realized many years ago that solar and stellar coronae are much hotter than the underlying photospheres. While the mechanisms responsible for coronal heating are not yet fully understood, it is clear from observations and modeling that magnetic fields play an important role in the heating process (Schrijver \& Zwaan 2000; Aschwanden 2004; Golub \& Pasachoff 2009). The energy is transported into the corona via magnetic structures in the lower atmosphere. In both active and quiet areas of the solar photosphere the magnetic field is highly fragmented (Stenflo 1973, 1989; Solanki 1993; Schrijver et al. 1998; Berger \& Title 2001; Priest et al. 2002; Abramenko \& Longcope 2005; DeWijn et al. 2009; Gonzalez et al. 2012). The field is concentrated into discrete flux elements with kilogauss field strengths and widths ranging from $100 \mathrm{~km}$ for the smallest observable elements to $3 \times 10^{4} \mathrm{~km}$ for large sunspots. In the corona above such regions the magnetic field is space filling, and the field strength is thought to be much more evenly distributed than in the photosphere below. Except directly over sunspots, the coronal field strength is likely to be well below $1 \mathrm{kG}$. Therefore, the flux tubes must expand with height in the photosphere and chromosphere to fill the available volume. Figure 1 shows a schematic view of the expanding flux tubes at one end of a coronal loop in an active region. As seen in emission at extreme ultraviolet (EUV) wavelengths the width of a coronal loop is typically $1000 \mathrm{~km}$ or larger (e.g., Aschwanden $\&$ Nightingale 2005), so one loop must be connected to multiple flux elements in the photosphere. At present the details of energy transport along these expanding flux elements are not yet fully understood. In this paper we explore some of the possibilities.

Theories of coronal heating can be divided into two main categories, stressing models and wave-heating models (Ionson 1985; Milano et al. 1997; Mandrini et al. 2000). In the magnetic stress or direct current (DC) heating models, the energy is extracted from coronal magnetic fields that are stressed by slow random footpoint motions. In wave or alternating current (AC) models, the heating results from the generation and dissipation of upwardly propagating waves. In both types of models the footpoint motions are due to interactions of granulescale convective flows with kilogauss magnetic flux elements in the photosphere. The key difference between DC and AC models is the timescale $\tau_{f}$ of the footpoint motions in comparison to the coronal Alfvén travel time $L_{\mathrm{cor}} / v_{A}$, where $L_{\mathrm{cor}}$ is the coronal loop length and $v_{A}$ the coronal Alfvén speed. When $\tau_{f} \gg L_{\mathrm{cor}} / v_{A}$, the corona has time to relax to an equilibrium state in which the various forces on the coronal plasma are nearly in balance. In this case the corona responds quasi-statically to the footpoint motions. For solar active regions this means that the coronal magnetic field is nearly force-free, $\nabla \times \mathbf{B} \approx \alpha \mathbf{B}$, where $\alpha$ is constant along field lines. In contrast, for $\tau_{f}<L_{\text {cor }} / v_{A}$ the corona responds in a wave-like manner to the footpoint motions (AC model).

In this context we define the timescale $\tau_{f}$ of the footpoint motions as follows. Let $\mathbf{u}(x, y, t)$ be the horizontal component of velocity at the base of the photosphere, i.e., the level where the continuum optical depth $\tau_{5000}=1$ at wavelength $\lambda=5000 \AA$. The velocity is a function of the horizontal coordinates $x$ and $y$, and of time $t$. The magnetic footpoints are carried by this horizontal flow, and follow paths $[x(t), y(t)]$ determined by the equations $d x / d t=v_{x}(t)=u_{x}(x, y, t)$ and $d y / d t=v_{y}(t)=$ $u_{y}(x, y, t)$. The correlation time $\tau_{c}$ of the footpoint motions is defined as the auto-correlation time of velocity $v_{x}(t)$ or $v_{y}(t)$. The dynamical time $\tau_{d}$ is defined as $\tau_{d} \equiv \ell_{\perp} / v_{\text {rms }}$, where $v_{\text {rms }} \equiv \sqrt{\left\langle v_{x}^{2}\right\rangle+\left\langle v_{y}^{2}\right\rangle}$ is the rms velocity, and $\ell_{\perp}$ is the autocorrelation length of the velocity field $\mathbf{u}(x, y, t)$ in the region where the magnetic footpoints are located. In this paper $\tau_{f}$ is defined as the shorter of the two timescales, $\tau_{f} \equiv \min \left(\tau_{c}, \tau_{d}\right)$, so a quasi-static response to the footpoint motions requires both $\tau_{c} \gg L_{\mathrm{cor}} / v_{A}$ and $\tau_{d} \gg L_{\mathrm{cor}} / v_{A}$. 


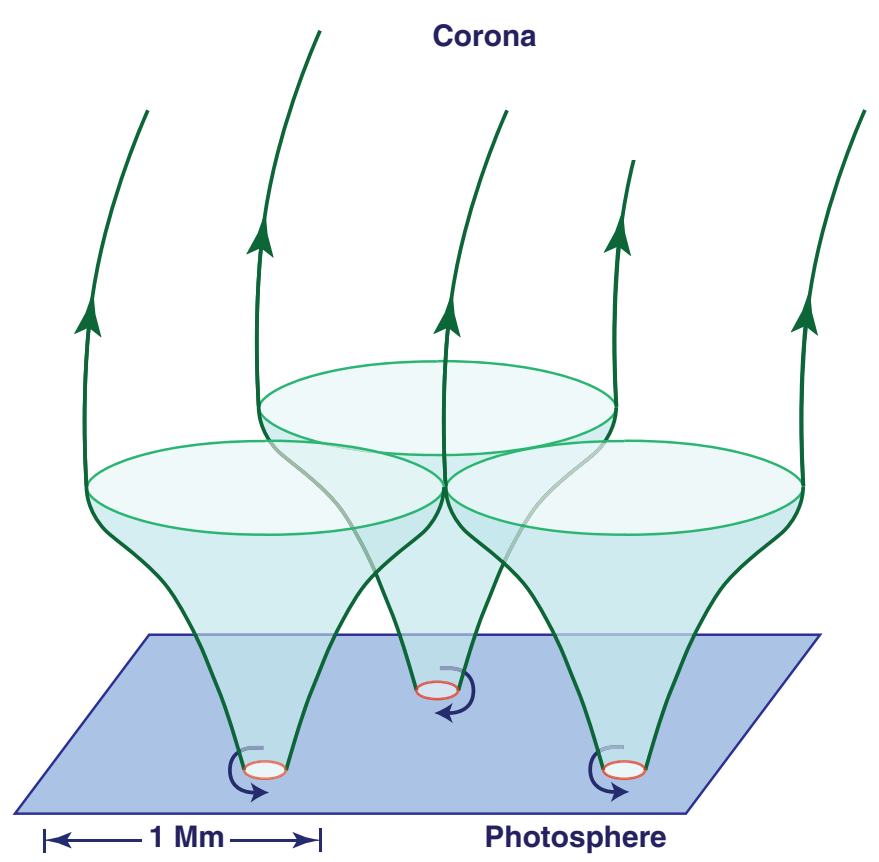

Figure 1. Schematic view of unipolar magnetic flux elements at one end of a coronal loop in a solar active region. The flux tubes expand with height in the photosphere, merge at height $z \sim 1 \mathrm{Mm}$ in the chromosphere (circles), and fill the available volume in the corona.

(A color version of this figure is available in the online journal.)

An example of the DC heating mechanism is the magnetic braiding model (Parker 1972, 1983; Berger 1993; Berger \& Asgari-Targhi 2009). The picture of coronal heating set out by Parker describes a highly conducting coronal plasma evolving due to slow, random footpoint motions. In this model it is assumed that the corona responds quasi-statically to the footpoint motions. Random walk of the footpoints causes tangling and braiding of the coronal field lines, and in ideal magnetohydrodynamics (MHD) the magnetic free energy builds quadratically with time (Berger 1993). Parker (1972) proposed that the complex topology of the braided field leads to the formation of tangential discontinuities (also see Ng \& Bhattacharjee 1998; Janse \& Low 2009; Low 2010; Janse et al. 2010; Craig 2010). These current layers may either burn slowly (e.g., tearing modes), or burn quickly in a series of reconnection events or "nanoflares" (Parker 1988). In some versions of the braiding model the reconnection switches on when the misalignment angle between neighboring flux tubes reaches a critical value (Parker 1988; Berger 1993; Dahlburg et al. 2005), and in other versions the reconnection occurs even for small angles (van Ballegooijen 1986). The response of coronal loops to nanoflares has been studied in great detail (e.g., Cargill \& Klimchuk 1997; Winebarger \& Warren 2005; Patsourakos \& Klimchuk 2006; Klimchuk et al. 2008; Reep et al. 2013), leading to a common view that solar active regions may be heated by nanoflare storms. Magnetic braiding may also determine the widths of coronal loops (Schrijver 2007).

Parker (1983) was the first to make detailed estimates of the heating rate in a braided field. He considered a simple model of a coronal loop, consisting of an initially uniform magnetic field between two parallel plates, which represent the photosphere at the two ends of the loop. Random motions imposed at the "photospheric" boundary plates cause small-scale twisting and braiding of the coronal field lines. Parker argued that in the absence of magnetic reconnection the transverse magnetic field $B_{t}$ increases linearly with time $t$ :

$$
B_{t}=B_{0} \tan \theta=B_{0} w t / L,
$$

where $B_{0}$ is the strength of the background coronal field, $w$ is the rms value of the footpoint velocity, and $L$ is the distance between the plates (loop length). The rate at which the footpoint motions do work on the field also increases linearly:

$$
F_{H}(t)=\frac{w B_{t} B_{0}}{4 \pi}=\frac{B_{0}^{2}}{4 \pi} \frac{w^{2} t}{L},
$$

which represents the energy flux into the corona. Parker assumed that once reconnection starts the energy input rate $F_{H}(t)$ saturates to a more constant value, and the time-averaged dissipation rate equals the time-averaged input rate. If dissipation is sufficiently slow that reconnection does not begin to destroy $B_{t}$ until it has accumulated for about 1 day, then a field strength of $100 \mathrm{G}$, a velocity of $0.4 \mathrm{~km} \mathrm{~s}^{-1}$, and a loop length of $100 \mathrm{Mm}$ yield an energy input rate $F_{H}=10^{7} \mathrm{erg} \mathrm{cm}^{-2} \mathrm{~s}^{-1}$, consistent with the observed rate for active regions (Parker 1983). The resulting pitch angles are quite large, $\theta \sim 20^{\circ}$.

Inherent in Parker's analysis is the assumption that coronal magnetic flux tubes can be wrapped around each other over a period of many hours, i.e., the flux tubes must retain their identity for a long time. This assumption is questionable because later observations have shown that photospheric flux elements continually split up and merge on a timescale of a few minutes (Berger \& Title 1996). After a flux element breaks up, the individual fragments disperse as a consequence of turbulent motions below the photosphere. If two or more neighboring flux elements break up, the individual fragments will move around with granular motion and will be swept into the edges of granules or supergranules. Eventually the fragments coalesce into new flux concentrations that will generally have a different mixture of flux fragments from the old flux elements. This process of splitting and merging will increase the topological complexity of the overlying coronal field (Figure 1). It is not clear what effect this will have on the process of coronal heating. Berger (1994) proposed that the increased complexity of the coronal field will contribute to the heating of the coronal loops. Another possibility is that the added complexity causes the magnetic free energy of the braided field to be released too early, which lowers the energy input rate (see Equation (2)); the predicted heating rate may then be insufficient to explain the observed heating (van Ballegooijen 1986). Therefore, at present it is unclear whether quasi-static braiding models can provide sufficient energy in active regions.

Numerical models of magnetic braiding have been developed by many authors. The modelers often followed Parker's approach whereby a coronal loop is approximated as an initially uniform field between two parallel plates, and the "footpoint" motions are applied at these boundary plates (e.g., Mikić et al. 1989; Longcope \& Strauss 1994; Hendrix et al. 1996; Galsgaard \& Nordlund 1996; Rappazzo et al. 2008; Wilmot-Smith et al. 2009a, 2009b). In effect, the photospheric footpoint motions are assumed to be directly transmitted to the coronal base without any change in velocity amplitude. Assuming footpoint motions with velocities of $1-2 \mathrm{~km} \mathrm{~s}^{-1}$ and timescales of 1-5 minutes (as observed), the numerical models predict that the corona tends to respond quasi-statically to the footpoint motions. However, such models ignore the fine structure of the photospheric magnetic field and the details of the magnetic coupling between the 
photosphere and corona over many density scale heights. Also, the assumption of a uniform background field makes it difficult to compare the predicted heating rates with observations.

Over the past decade, more realistic three-dimensional (3D) MHD models of active regions have been developed (e.g., Gudiksen \& Nordlund 2002, 2005a, 2005b; Bingert \& Peter 2011, 2013; Peter \& Bingert 2012; Bourdin et al. 2013). These models include the effects of the lower atmosphere, and are based on observed magnetograms, making the lower boundary conditions of the model much more realistic. The photospheric driver includes incompressible flows that match the statistical properties of the solar granulation (Gudiksen \& Nordlund 2002). The models predict that the corona responds almost quasistatically to the footpoint motions, and that the coronal heating is dominated by the dissipation of field-aligned electric currents (e.g., Bingert \& Peter 2011). At present the spatial resolution of such models is still relatively poor $(\sim 200 \mathrm{~km})$, and the models do not resolve the fine structure of the photospheric field. We speculate that as the resolution of the models is further increased, the magnetic perturbations may become more wave-like and the heating may be dominated by $\mathrm{AC}$ processes.

The modeling by Gudiksen \& Nordlund (2005a) and others is a major step forward, but it is not clear that the models have obtained the required coronal heating rates. For example, in the simulation by Bingert \& Peter (2011) the energy flux into the corona is only about $10^{5} \mathrm{erg} \mathrm{cm}^{-2} \mathrm{~s}^{-1}$ (see Figure 8 of their paper), and the horizontally averaged coronal temperature is less than $1 \mathrm{MK}$ (their Figure 3), whereas observed active regions have energy fluxes of about $10^{7} \mathrm{erg} \mathrm{cm}^{-2} \mathrm{~s}^{-1}$ (Withbroe $\&$ Noyes 1977) and temperatures in the range 3-5 MK. In thermal equilibrium the heating rate $Q$ must roughly equal the energy loss rate, which for hot loops is dominated by thermal conduction and scales as $Q \propto T_{\max }^{7 / 2}$, where $T_{\max }$ is the peak temperature in the loop. Therefore, to boost the temperature from $1 \mathrm{MK}$ to $3 \mathrm{MK}$ requires a significant increase in the heating rate (by a factor $\sim 47$ ). It is not clear that DC models can explain how footpoint motions with velocities of $1-2 \mathrm{~km} \mathrm{~s}^{-1}$ can produce sufficient heating. This difficulty was noticed already in an early version of the DC heating model (van Ballegooijen 1986), where the footpoint motions were constrained by observed rates of magnetic flux dispersal in the photosphere. The basic problem is that in the DC model the transverse velocities at the coronal base are similar to the those in the photosphere (only a few $\mathrm{km} \mathrm{s}^{-1}$ ), but much higher velocities are needed to heat the corona to temperatures in the range $3-5 \mathrm{MK}$.

The wave heating model also has a long history. Alfvén waves have received particular attention because of their ability to transport energy over different layers of the solar atmosphere (e.g., Coleman 1968; Uchida \& Kaburaki 1974; Wentzel 1974; Hollweg et al. 1982). Different mechanisms for the dissipation of the Alfvén waves have been proposed. These mechanisms involve either phase mixing and resonant absortion (Heyvaerts \& Priest 1983; De Groof \& Goossens 2002; Goossens et al. 2011), turbulent cascade of wave energy (e.g., Hollweg 1986; Gómez \& Ferro Fontán 1988; Heyvaerts \& Priest 1992; Milano et al. 1997; Chae et al. 1998; Matthaeus et al. 1999; Verdini \& Velli 2007; Cranmer et al. 2007), or coupling with compressive wave modes (Kudoh \& Shibata 1999; Moriyasu et al. 2004; Suzuki \& Inutsuka 2006; Antolin et al. 2008; Antolin \& Shibata 2010; Matsumoto \& Shibata 2010). The Alfvén wave turbulence (AWT) model was first proposed for the solar wind, but has also been applied to coronal loops (van Ballegooijen et al.
2011; Asgari-Targhi \& van Ballegooijen 2012; Asgari-Targhi et al. 2013, hereafter papers I, II, and III). According to this model, counter-propagating Alfvén waves interact nonlinearly with each other, producing an anisotropic turbulent cascade of wave energy to small spatial scales, where the energy is dissipated.

In paper I a reduced MHD model for AWT in coronal loops was developed. The Alfvén waves are driven by footpoint motions that occur on very small spatial scales in the photosphere (less than $100 \mathrm{~km}$ ). The motions are assumed to occur inside kilogauss flux elements, and are due to the interactions of these flux elements with convective flows in their local surroundings. The footpoint velocities are assumed to be $1-2 \mathrm{~km} \mathrm{~s}^{-1}$ with correlation times $\tau_{c}$ in the range 30-100 s, similar to the values measured for the proper motions of the flux elements (Chitta et al. 2012). It was found that the magnetic field at larger heights along the flux tube responds dynamically to these footpoint motions, and is driven far from the force-free equilibrium state. As discussed in papers I and II, the AWT model can reproduce several key observations of active region loops.

Several authors have used observations to test models of solar coronal heating. Mandrini et al. (2000) used observations of coronal loops in soft X-rays, and found that models involving the gradual stressing of magnetic fields are generally in better agreement with the observations than are wave heating models (also see Démoulin et al. 2003). Schrijver \& Aschwanden (2002) and Schrijver et al. (2004) simulated the appearance of the solar corona in various wavelength bands, using different assumed heating mechanisms. The best match to the X-ray and EUV observations was obtained for an energy flux $F_{H}$ into the corona given by $F_{H} \approx 4 \times 10^{14} \mathrm{~B} / L_{h}$ (in $\mathrm{erg} \mathrm{cm}^{-2} \mathrm{~s}^{-1}$ ), where $B$ is the magnetic field strength at the chromospheric base (in G) and $L_{h}$ is the loop half-length. Based on this $B / L_{h}$ scaling, Schrijver et al. (2004) concluded that DC reconnection at tangential discontinuities is the most likely mechanism for coronal heating. However, this modeling did not describe in detail how the energy is injected into the corona.

In this paper we further investigate whether photospheric footpoint motions with velocities of $1-2 \mathrm{~km} \mathrm{~s}^{-1}$ can heat the corona in active regions, and whether the corona responds quasistatically or dynamically to such footpoint motions (DC versus AC heating). Both Alfvén waves and quasi-static perturbations of the magnetic field are considered, and are treated in a common framework by solving the 3D reduced MHD equations for a thin flux tube that extends from one end of a coronal loop to the other end. Two different models for the magnetic field are considered. In the first model only the coronal part of the loop is considered, and the boundary conditions are applied at the height of the chromosphere-corona transition region (TR), similar to the approach used by Parker (1972), Mikić et al. (1989), and others. The second (more realistic) model includes the lower atmosphere, and the footpoint motions are applied at the base of the photosphere. However, even this second model still makes an important approximation: only a single magnetic flux tube is considered, i.e., we do not include the effects of multiple flux components (Figure 1). The reason is that the interactions of multiple flux elements separated by field-free plasma cannot be modeled using the "reduced" MHD equations. Such modeling would require solving the full MHD equations for the stratified atmosphere in which the flux tubes are embedded, with enough spatial resolution to simulate the dynamics of Alfvén waves. This task is very challenging and is beyond the scope of this paper. Our current approach is based on the assumption that the 
energy transport into the corona is dominated by waves (or other disturbances) that propagate along individual flux elements, and that the interactions between neighboring flux elements play only a secondary role in the coronal heating process. We find that for a given footpoint velocity the second model behaves much more dynamically and produces much more heating than the first model. The results of this study are important for understanding whether the heating on the Sun is dominated by AC or DC processes, and to test the models against observations.

The paper is organized as follows. In Section 2 the features common to the two MHD models are described, including the properties of the background atmosphere and shape of the magnetic flux tube. In Sections 3 we present the results for the first model, in which the footpoint motions are applied at the height of the TR, and in Section 4 we present results for the more realistic model where footpoint motions are applied in the photosphere. The results are further discussed in Section 5.

\section{MHD MODELS}

In this paper we simulate the dynamics of plasma and magnetic field inside a coronal loop, using the so-called reduced MHD model (e.g., Strauss 1976, Paper I). Two different versions of the MHD model will be used. In version 1 only the coronal part of the loop is considered, and the two end points of the modeled flux tube are located at the TR, which is taken to be the height where the temperature $T \approx 2 \times 10^{4} \mathrm{~K}$. This version of the model is illustrated in Figure 2(a). The TRs at the two ends of the coronal loop lie at heights of about $2 \mathrm{Mm}$ above the photosphere, and are indicated by two red circles in the figure. The radius of the circles is $1 \mathrm{Mm}$ so the coronal tube is connected to several flux tubes in the lower atmosphere. As the magnetic field evolves due to random motions of the photospheric footpoints, the magnetic flux elements in the lower atmosphere (green tubes) are assumed to remain nearly vertical, i.e., the footpoint motions are assumed to be directly transmitted from the photosphere to the TR without much change in velocity or timescale of the motions. Hence, in version 1 the coronal flux tube encompasses several photospheric flux tubes (Figure 1), but the details of the photospheric flux tubes are ignored and "footpoint" motions are in fact applied directly at the height of the TR. We will model the input of braiding structure into a coronal loop using a continuous motion at the TR. Such motions can provide a significant amount of braiding structure, as seen in this study and in others (Wilmot-Smith et al. 2009b).

In contrast, in version 2 of the MHD model only a single magnetic flux tube is considered. The tube extends from the photosphere at one end of the coronal loop, through the chromosphere and corona, to the photosphere at the other end, as shown in Figure 2(b). The radius of the flux tube in the photosphere is $100 \mathrm{~km}$ and is indicated by the red circles. At the height of the TR the radius of the flux tube is about $400 \mathrm{~km}$, less than that in Figure 2(a). In this case the footpoint motions are applied at the photospheric base $\left(\tau_{5000}=1\right)$, and Alfvén waves are launched into the flux tube. Version 2 is the AWT model described in papers I, II, and III. Version 2 does not include the effects of multiple flux components (Figure 1), but does include the effects of density stratification in the lower atmosphere, which are important for accurately simulating the dynamics of Alfven waves.

Version 1 is somewhat unrealistic in that it ignores the details of the magnetic coupling between the photosphere and corona. It is indeed not realistic to assume that the flux tubes remain vertical in the lower atmosphere, and that the footpoint motions
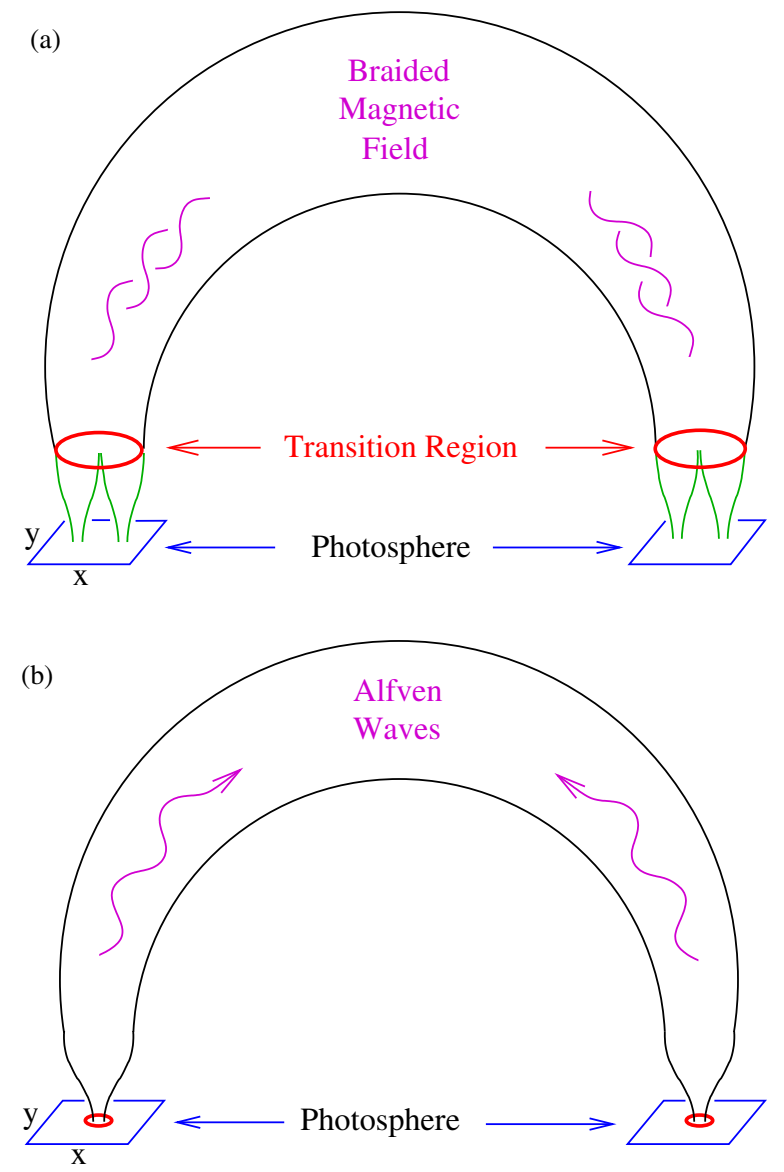

(c)

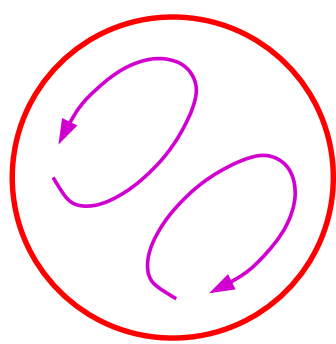

Figure 2. Two versions of the reduced MHD model, and imposed footpoint motions. (a) Version 1 in which only the coronal part of the loop is modeled, and boundary conditions are imposed at the TRs, leading to DC heating. (b) Version 2 in which the entire loop is modeled, and boundary conditions are imposed at the photosphere, leading to AC heating. (c) In both cases the footpoint motions have a velocity pattern consisting of two counter-rotating cells. The velocity stream function $f(x, y, t)$ is a superposition of two modes that depend on $\cos \varphi$ and $\sin \varphi$, respectively, where $\varphi$ is the azimuth angle (see paper I for details).

(A color version of this figure is available in the online journal.)

are directly transmitted into the corona. However, there are two reasons for including such models in our study of coronal heating. First, it allows us to reproduce results from earlier studies (e.g., Mikić et al. 1989; Rappazzo et al. 2007, 2008) in which similar approximations were made. Second, we find that in version 2 of the model there is a much more dynamic response to the footpoint motions compared to version 1 , and so far we have not been able to produce a realistic DC heating using version 2 . Therefore, version 1 is needed to demonstrate that the nature of the magnetic perturbations (Alfvén waves versus quasi-static evolution) depends strongly on the way the lower atmosphere is treated in the model, and that a DC response can be obtained by ignoring the lower atmosphere altogether. 
In both versions of the MHD model the flux tube is assumed to have a circular cross-section with radius $R(s)$, where $s$ is the distance along the tube $(s=0, \cdots, L$, where $L$ is the total length of the tube). The overall curvature of the loop is neglected, so the background magnetic field $\mathbf{B}_{0}(x, y, s)$ is a function of the cartesian coordinates $x, y$, and $s$. The magnetic field strength $B_{0}(s)$ and plasma density $\rho_{0}(s)$ are assumed to be nearly constant over the loop cross-section. The magnetic flux is constant along the tube, so that $R(s) \propto\left[B_{0}(s)\right]^{-1 / 2}$. We use a numerical approach where the imposed footpoint motions are confined to a circular area, $x^{2}+y^{2} \leqslant R_{\text {base }}^{2}$, where $R_{\text {base }}[=R(0)$ or $R(L)]$ is the tube radius at the TR (Version $1, R_{\text {base }}=1 \mathrm{Mm}$ ) or in the photosphere (version $2, R_{\text {base }}=100 \mathrm{~km}$ ). The motions within this circular area are assumed to be incompressible and transverse to the flux tube axis. The pattern of motion consists of two counter-rotating cells with arbitrary orientation, as illustrated in Figure 2(c). This pattern is a superposition of two modes with azimuthal wave number $m=1$ (see paper I for details).

The mode amplitudes change randomly with time. Such random time series are constructed by creating a sequence of (normally distributed) random numbers, and Fourier filtering the sequence using a Gaussian function $G(\tilde{v})=\exp \left[-\left(\tau_{0} \tilde{v}\right)^{2}\right]$, where $\tilde{v}$ is the temporal frequency and $\tau_{0}$ is a specified model parameter. In previous work we described $\tau_{0}$ as the "correlation time" but we now realize this is not quite correct. Here we define the correlation time as

$$
\tau_{c} \equiv \int_{0}^{\infty} C(\Delta t) d \Delta t
$$

where $\Delta t$ is the time delay between two measurements, and $C(\Delta t)$ is the correlation between the measurements, which is normalized such that $C(0)=1$. Then our random sequences have a correlation time $\tau_{c}=\tau_{0} / \sqrt{2 \pi}$. The same correction may be applied to the correlation times listed in papers I, II, and III.

In the reduced MHD approximation the slow and fast MHD modes are filtered out, and only the Alfvén mode is retained. Only the magnetic and velocity fluctuations are simulated, and flows along the background field are neglected. The magnetic fluctuations are assumed to be small compared to the background field, and are approximated as $\mathbf{B}_{1}=\nabla_{\perp} h \times \mathbf{B}_{0}$, where $h(x, y, s, t)$ is the magnetic flux function and $t$ is the time. Similarly, the velocity fluctuations are approximated as $\mathbf{v}_{1}=$ $\nabla_{\perp} f \times \hat{\mathbf{B}}_{0}$, where $f(x, y, s, t)$ is the velocity stream function and $\hat{\mathbf{B}}_{0}$ is the unit vector along the background field. We also define the parallel component of vorticity, $\omega(x, y, s, t) \equiv-\nabla_{\perp}^{2} f$, and the magnetic torsion parameter, $\alpha(x, y, s, t) \equiv-\nabla_{\perp}^{2} h$. The functions $f$ and $h$ satisfy the following differential equations (paper I):

$$
\begin{gathered}
\frac{\partial \omega}{\partial t}=-[\omega, f]+v_{A}^{2}\left\{\hat{\mathbf{B}}_{0} \cdot \nabla \alpha+[\alpha, h]\right\}+D_{v}, \\
\frac{\partial h}{\partial t}=\hat{\mathbf{B}}_{0} \cdot \nabla f+\frac{f}{H_{B}}+[f, h]+D_{m},
\end{gathered}
$$

where $H_{B}(s) \equiv B_{0} /\left(d B_{0} / d s\right)$ is a magnetic scale length, and $[\cdots, \cdots]$ is the bracket operator, which involves derivatives in $x$ and $y$. To evaluate these derivatives we use a spectral method with maximum wavenumber $k_{\perp, \max }=30 / R(s)$. The terms $D_{v}$ and $D_{m}$ describe the effects of an artificial viscosity and resistivity (see papers I and III for details).

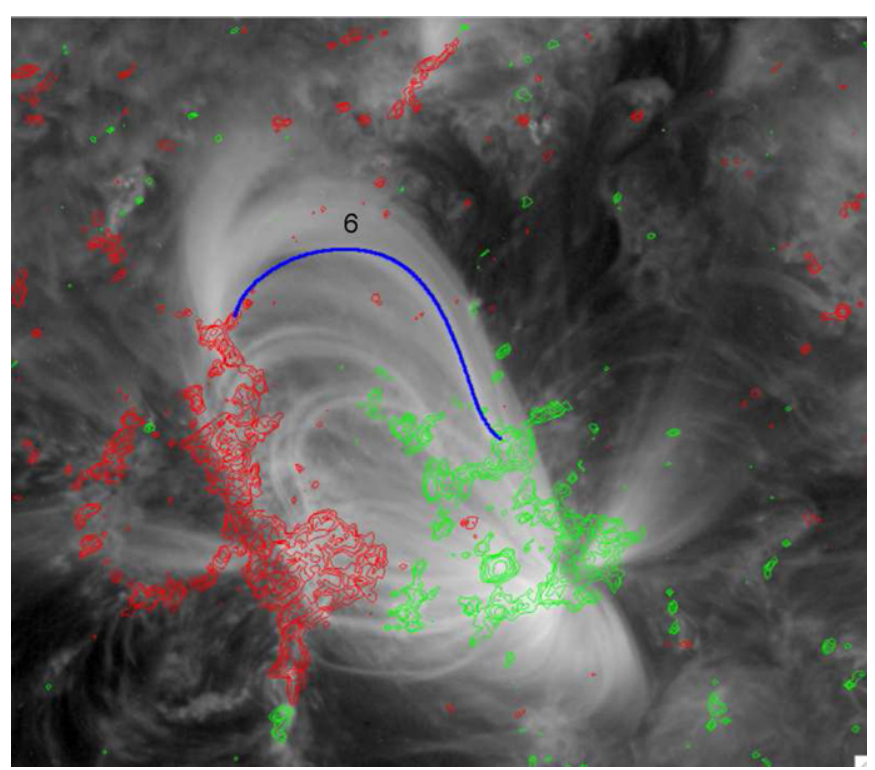

Figure 3. SDO observation of active region NOAA 11067 on 2010 May 5 at 3:00 UT with field of view $276 \times 276 \mathrm{Mm}$. The field line is traced through the NLFFF model. The background image is from the AIA $171 \AA$ A channel. Red and green contours show the photospheric magnetic flux distribution based on the HMI magnetogram.

(A color version of this figure is available in the online journal.)

In our model the free energy is dissipated via a turbulent cascade. The 3D turbulence is explicitly simulated, albeit with relatively low spatial resolution $\sim 0.1 R(s)$. The viscous and resistive damping rates only affect the highest wavenumbers of the turbulent cascade, and the energy cascade rate depends weakly on the values of the viscous and resistive dissipation coefficients. In this work we use hyperdiffusion with damping rates depending on the fourth power of the perpendicular wavenumber $k_{\perp}$ (same as in paper III). The damping rate $\gamma$ is given by

$$
\gamma\left(s, t, k_{\perp}\right)=70 \frac{v_{\mathrm{rms}}(s, t)}{R(s)}\left[\frac{k_{\perp}}{k_{\perp, \max }(s)}\right]^{4},
$$

where $v_{\text {rms }}(s, t)$ is the rms velocity of the perpendicular motions (time averaged over a time interval of $300 \mathrm{~s}$ ). These parameters vary strongly with position along the loop.

In paper II we developed a 3D magnetic model for active region NOAA 11067, which was observed on 2010 May 5 at 3:00 UT with instruments on the Solar Dynamics Observatory $(S D O)$ satellite. The nonlinear force-free field (NLFFF) model is based on magnetograms from the Helioseismic and Magnetic Imager (HMI) on SDO. In paper II we selected field lines traced through the magnetic model and simulated the Alfvén waves in thin flux tubes surrounding those field lines. Here we consider one of these flux tubes, labeled 6 in paper II. Figure 3 shows the projection of the flux tube axis (blue curve) projected onto the plane of sky and overplotted on an image of the active region. The image was obtained with the Atmospheric Imager Assembly (AIA) on SDO. In the next two sections we present models for the heating inside this flux tube, using versions 1 and 2 of the MHD code. All models use essentially the same background atmosphere with a coronal base pressure $p_{\text {cor }}=1.0$ dyne $\mathrm{cm}^{-2}$ and maximum temperature $T_{\max }=2.45 \mathrm{MK}$. The TR heights at the two ends of the loop are $z_{\mathrm{TR} 1}=2628 \mathrm{~km}$ and $z_{\mathrm{TR} 2}=2605 \mathrm{~km}$. The field strength $B_{0}(s)$ varies along the flux tube, and its minimum value in 

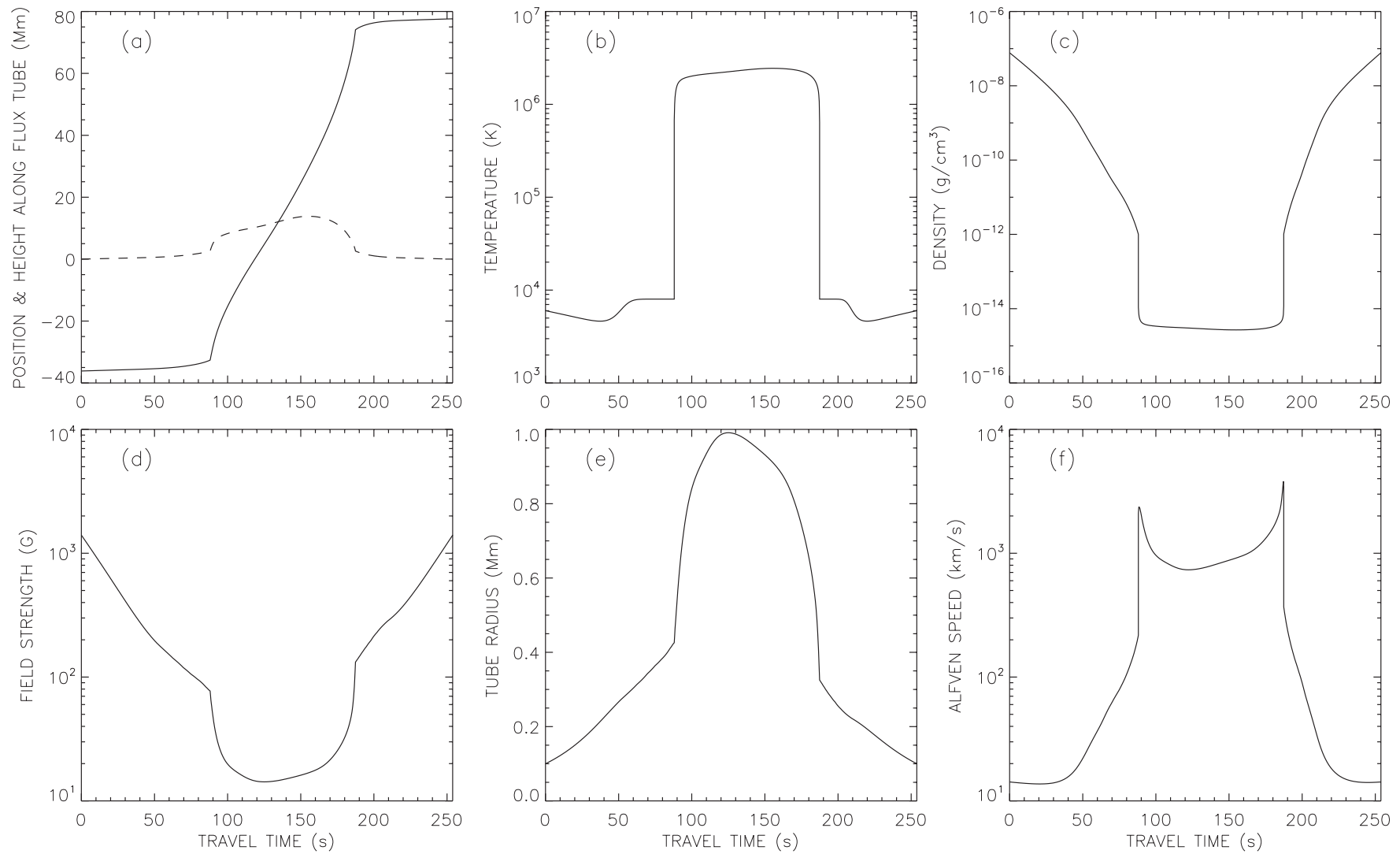

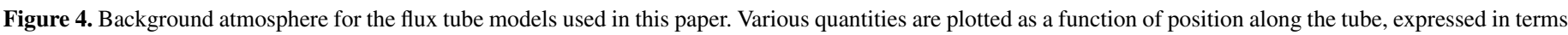

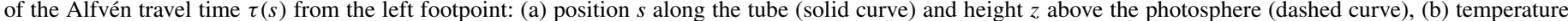

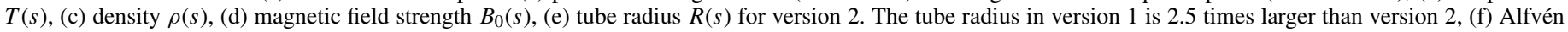

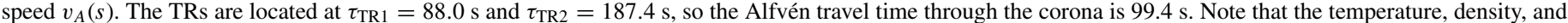
Alfvén speed are discontinuous at the TRs.

Table 1

Model Parameters

\begin{tabular}{lcrcccc}
\hline \hline Model & $\begin{array}{c}v_{\text {rms }} \\
\left(\mathrm{km} \mathrm{s}^{-1}\right)\end{array}$ & \multicolumn{1}{c}{$\begin{array}{c}\tau_{c} \\
(\mathrm{~s})\end{array}$} & $\begin{array}{c}Q_{\min } \\
\left(\mathrm{erg} \mathrm{cm}^{-3} \mathrm{~s}^{-1}\right)\end{array}$ & $m$ & $\begin{array}{c}p_{\text {pred }} \\
\left(\text { dyne cm }^{-2}\right)\end{array}$ & $\begin{array}{c}T_{\text {pred }} \\
(\mathrm{MK})\end{array}$ \\
\hline M1 & 1.48 & 239.4 & $6.21 \times 10^{-6}$ & 0.689 & 0.047 & 0.73 \\
M2 & 1.48 & 23.9 & $6.27 \times 10^{-4}$ & 0.712 & 1.36 & 2.10 \\
M3 & 1.48 & 398.9 & $1.55 \times 10^{-4}$ & 0.505 & 0.48 & 1.51 \\
M4 & 0.10 & 398.9 & $5.57 \times 10^{-7}$ & 0.739 & 0.006 & 0.38 \\
M5 & 1.48 & 23.9 & $1.94 \times 10^{-4}$ & 0.680 & 0.58 & 1.57 \\
\hline
\end{tabular}

the corona $B_{\min }=14.3 \mathrm{G}$. The field strengths at the two TRs are $B_{\mathrm{TR} 1}=76.7 \mathrm{G}$ and $B_{\mathrm{TR} 2}=124.7 \mathrm{G}$, so the mean loop expansion factor is $\Gamma \equiv(1 / 2)\left(B_{\mathrm{TR} 1}+B_{\mathrm{TR} 2}\right) / B_{\min } \approx 7.1$. The coronal loop length is $L_{\text {cor }}=106.3 \mathrm{Mm}$ (Figure 3$)$. The model takes into account the $3 \mathrm{D}$ shape of the coronal loop as derived from the NLFFF model and includes the effect of stratification.

Figure 4 shows various background quantities plotted as function of position along the flux tube. Positions are expressed in terms of the Alfvén travel time from the left footpoint, $\tau(s) \equiv$ $\int_{0}^{s} d s / v_{A}(s)$. Note that the Alfvén speed $v_{A}(s)$ varies strongly with position along the tube (see Figure 4(f)). All models used in this paper have the same background atmosphere, except that version 1 only uses the coronal part of the loop. The model parameters are listed in Table 1. The first column gives the model name, and the second and third columns give the velocity and correlation time of the footpoint motions, $v_{\mathrm{rms}}$ and $\tau_{c}$.

\section{MODEL WITH BOUNDARY CONDITIONS AT THE TRANSITION REGION}

In this section we use version 1 of the MHD code, in which only the coronal part of the loop is considered and the "footpoint" motions are imposed at the TR. Model M1 is based on the somewhat unrealistic assumption that in the lower atmosphere the flux tubes remain vertical as they are moved about by the convective flows. The top panels in Figure 5 show the vorticities $\omega_{k}(t)$ of the two driver modes used in this model: $k=9$ for the mode with velocity stream function $f \propto \cos \varphi$ (full curve), and $k=10$ for the mode with $f \propto \sin \varphi$, where $\varphi$ is the azimuth angle relative to the flux tube axis. Figures 5(a) and (b) show the vorticities as function of time for the left and right footpoints, respectively. The imposed random footpoint motions have a velocity amplitude $v_{\mathrm{rms}}=1.48 \mathrm{~km} \mathrm{~s}^{-1}$ and correlation time $\tau_{c}=600 / \sqrt{2 \pi}=239.4 \mathrm{~s}$, typical for the observed random motions of the underlying photosphere. This corresponds to a diffusion constant $D=(1 / 2) v_{\mathrm{rms}}^{2} \tau_{c}=262 \mathrm{~km}^{2} \mathrm{~s}^{-1}$, similar to the value found by DeVore et al. (1985). The parameters for model M1 are listed in Table 1.

We simulated the dynamics of magnetic fields inside the coronal loop by solving the 3D reduced MHD Equations (4) and (5). The imposed footpoint motions cause twisting and braiding of coronal field lines, and build-up of field-aligned electric currents. The evolution of the magnetic field in model M1 was simulated for a period of $12,000 \mathrm{~s}$ with time step $\Delta t=0.5 \mathrm{~s}$. Figure 5(c) shows the energy density $E(t)$ of the braided field as a function of time. This energy density is an 

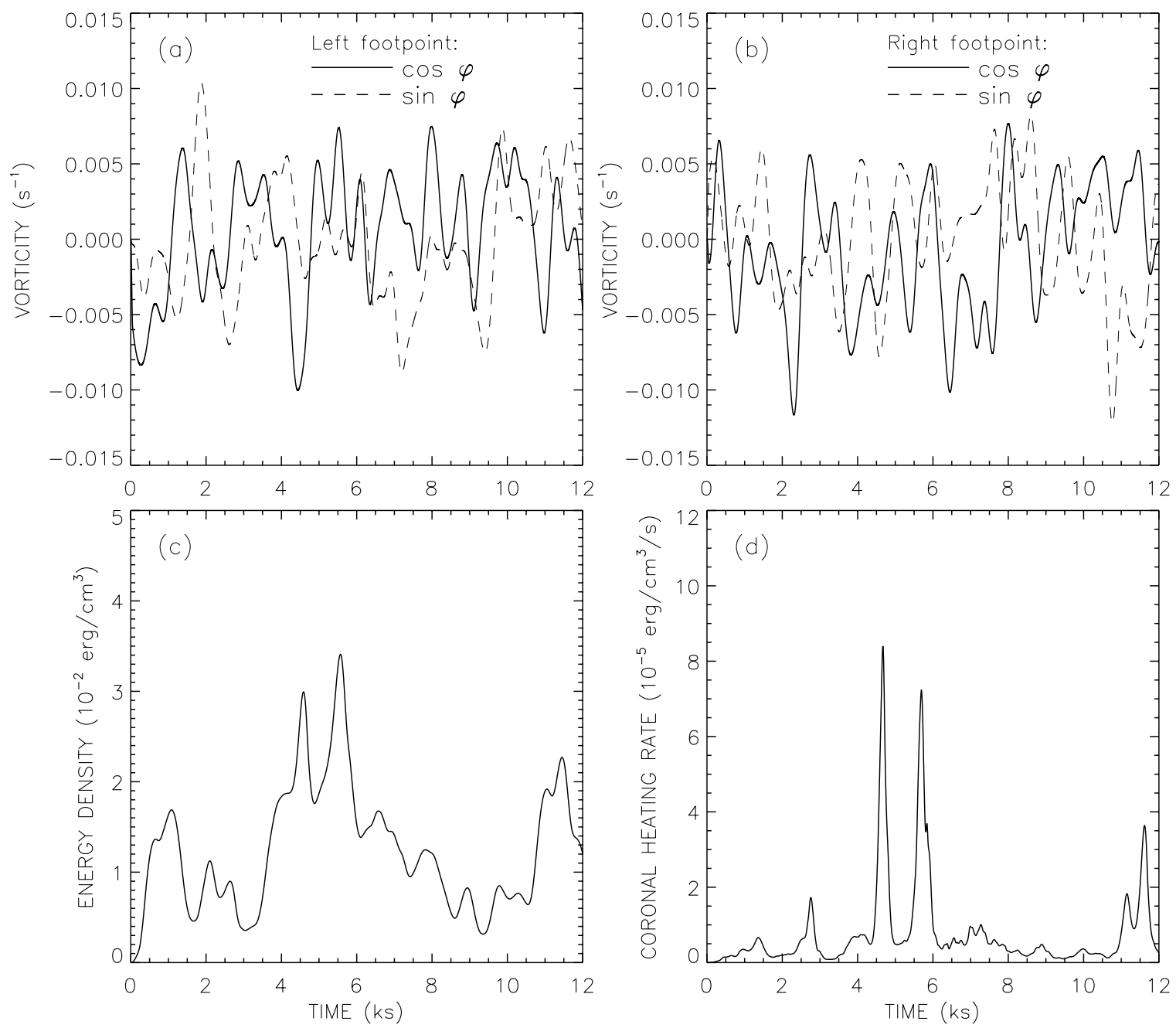

Figure 5. Results for model M1 in which only the coronal part of the loop is simulated, and "footpoint" motions are applied at the TRs. The top panels show the vorticities $\omega_{k}(t)$ of the two driver modes that make up the velocity pattern (see Figure 2(c)). Full and dashed curves are for modes that depend on $\cos \varphi$ and $\sin \varphi$, respectively, where $\varphi$ is the azimuth angle. Panel (a) shows the vorticities for the left footpoint $(s=0)$, panel (b) for the right footpoint $(s=L)$. The imposed motions have a velocity amplitude $v_{\mathrm{rms}}=1.48 \mathrm{~km} \mathrm{~s}^{-1}$ and correlation time $\tau_{c}=239.4 \mathrm{~s}$. (c) Energy density $E(t)$ of the braided field in the corona. (d) Coronal heating rate $Q(t)$.

average over the coronal volume. The energy dissipation rate $Q(t)$ is shown in Figure 5(d), and is also a volume average. Note that there are two bursts of heating at times $t \approx 4700 \mathrm{~s}$ and $t \approx 5700 \mathrm{~s}$. These bursts are associated with reconnection events in the current sheet that forms in this model. The peak heating rates in these events are $Q_{\text {peak }} \approx 8 \times 10^{-5} \mathrm{erg} \mathrm{cm}^{-3} \mathrm{~s}^{-1}$.

Figure 6 shows various quantities plotted as function of position along the loop for model M1. All these quantities are averaged over the loop cross-section, and over time (last 11,800 s of the simulation). Positions along the loop are expressed in terms of the Alfvén travel time $\tau(s)$ from the left TR. Figure 6(a) shows the energy density of the magnetic fluctuations (dashed curve), the kinetic energy density of the velocity fluctuation (dotted curve), and the total energy density (full curve). Note that the magnetic free energy is much larger than the kinetic energy, i.e., the free energy is dominated by the magnetic field. This means that the magnetic configuration is near an equilibrium state and since gas pressure effects are not included in our RMHD modeling, the perturbed field is force-free; $\mathbf{J} \times \mathbf{B} \approx 0$. Similarly, Figure 6(d) shows the energy dissipation rates due to the viscous and resistive terms in the Equations (4) and (5). The total dissipation rate $Q(s, t)$ is assumed to be equal to the heating rate of the coronal plasma. We find that for model M1 the time-averaged heating rate is quite low; near the loop top $Q \approx 6.21 \times 10^{-6} \mathrm{erg} \mathrm{cm}^{-3} \mathrm{~s}^{-1}$.

The middle and right panels in Figure 6 show the rms values of velocity, vorticity, magnetic field fluctuation, and twist parameter $\alpha_{\text {rms }}$. Note that $\alpha_{\text {rms }}(s)$ is nearly constant, again indicating that the magnetic field is nearly force-free. Therefore, in model M1 the corona responds quasi-statically to the "footpoint" motions, and the heating in this model can be classified as a DC heating mechanism.

The time-averaged heating rate $Q(s)$ varies with position along the coronal loop, and can be then fitted by a power law:

$$
Q(s)=Q_{\min }\left[\frac{B(s)}{B_{\min }}\right]^{m},
$$

where $B(s)$ is the magnetic field strength along the loop, $B_{\min }$ and $Q_{\min }$ are the minimum values of field strength and heating rate in the corona, and $m$ is an exponent. The quantities $Q_{\text {min }}$ and $m$ are determined by the fit, and are listed in the fourth and fifth columns of Table 1 .

To estimate the effect of the heating on coronal pressure and temperature, we also compute the temperature $T(s)$ and pressure $p(s)$, using a one-dimensional loop modeling code 

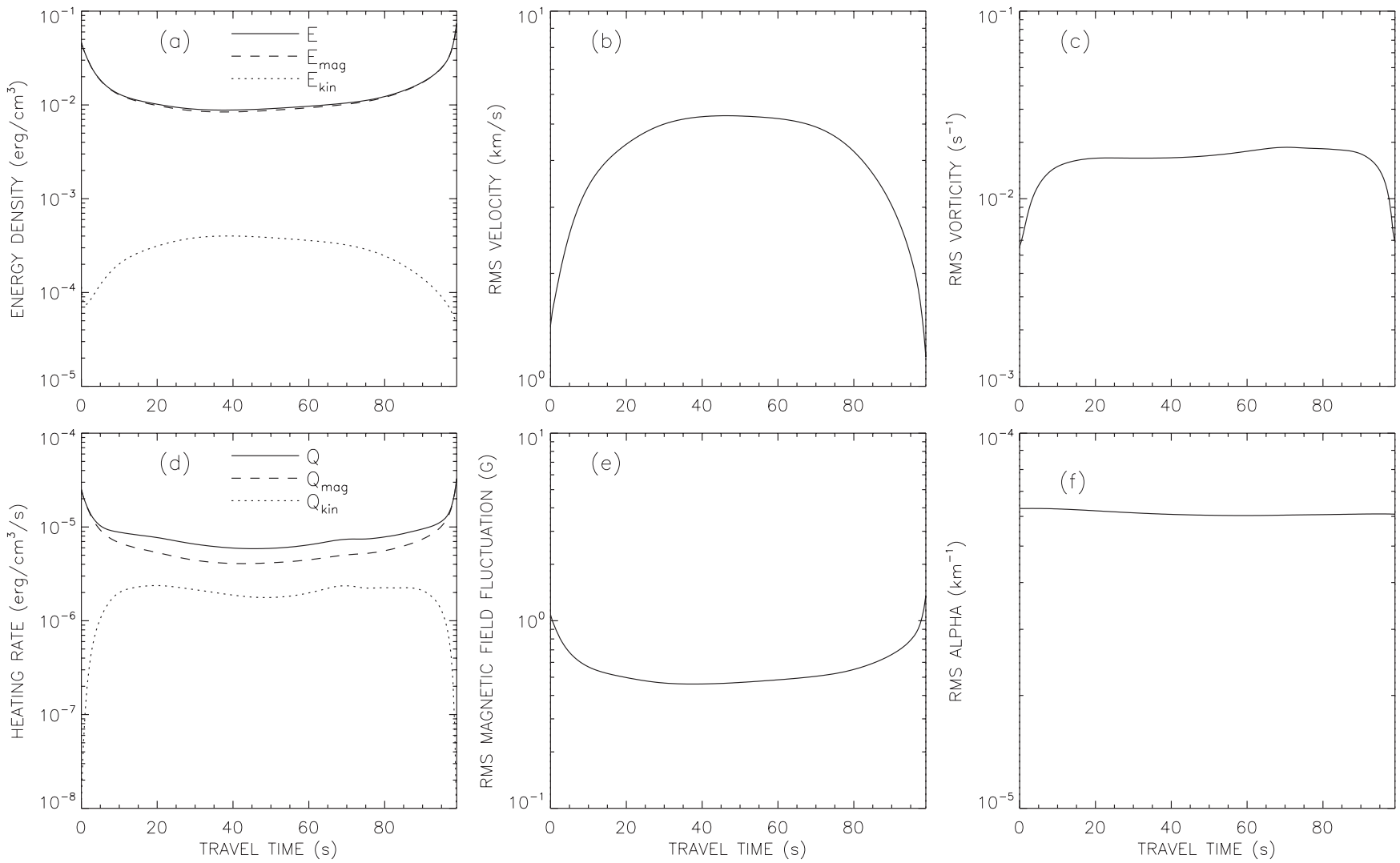

Figure 6. Various quantities plotted as function of position along the loop for model M1: (a) energy density $E(s)$ (solid curve) and contributions from kinetic and magnetic energy, (b) velocity amplitude, (c) parallel component of vorticity, (d) energy dissipation rate $Q(s)$ (full curve) and contributions from kinetic and magnetic energy dissipation, (e) transverse magnetic field fluctuation, (f) parameter $\alpha_{\mathrm{rms}}(s)$ describing the twist of magnetic field lines. These quantities are plotted as a function of Alfvén travel time $\tau(s)$ from the left transition region. All quantities have been averaged over the cross-sectional area of the loop and over time. Panels (b)-(f) give the rms values of the relevant parameters. The plasma heating rate is assumed to be equal to the total dissipation rate $Q(s)$ shown in panel (d).

(see Schrijver \& van Ballegooijen 2005). This code solves the energy transport equations for a coronal loop with arbitrary field strength $B(s)$, and includes the effects of coronal heating, thermal conduction, enthalphy flux, and optically thin radiative losses. The heating is assumed to be steady in time and is given by Equation (7). For model M1 the predicted coronal base pressure $p_{\text {pred }}=4.7 \times 10^{-2}$ dyne $\mathrm{cm}^{-2}$, and the predicted peak temperature $T_{\text {pred }} \approx 0.73 \mathrm{MK}$ (see Table 1 ). This pressure is so low that it does not make sense to further iterate between the models as we did in paper II. Note that the pressure is much lower than the value used in the model setup $\left(1 \mathrm{dyne} \mathrm{cm}^{-2}\right)$, so model M1 is not in thermal equilibrium (heating is not balanced by radiative and conductive losses) and the above values for $p_{\text {pred }}$ and $T_{\text {pred }}$ are only crude estimates. Nevertheless, it is clear that the predicted coronal pressure and temperature are much lower than what is needed for active region loops, which have temperatures in the range 3-5 MK.

We studied the evolution of the magnetic field in model M1 by randomly selecting 50 points in the loop cross-section at position $s=0.44 \mathrm{~L}$ and time $t=4000 \mathrm{~s}$, and following these particles in time assuming they are passively advected by the flow. For each time $t$ we trace out the 50 field lines that pass through these points, and plot their shapes in a perspective view. Figure 7 shows the resulting shapes at time $t=6000 \mathrm{~s}$. Note that the field lines are twisted and braided about each other.

An animation of Figure 7 is available in the online journal. This animation covers the period $4000 \leqslant t \leqslant 6000$, and shows that the magnetic field usually evolves slowly with time, but somewhat faster evolution occurs during the two energy release events at $t \approx 4700 \mathrm{~s}$ and $t \approx 5700 \mathrm{~s}$. However, it should be noted

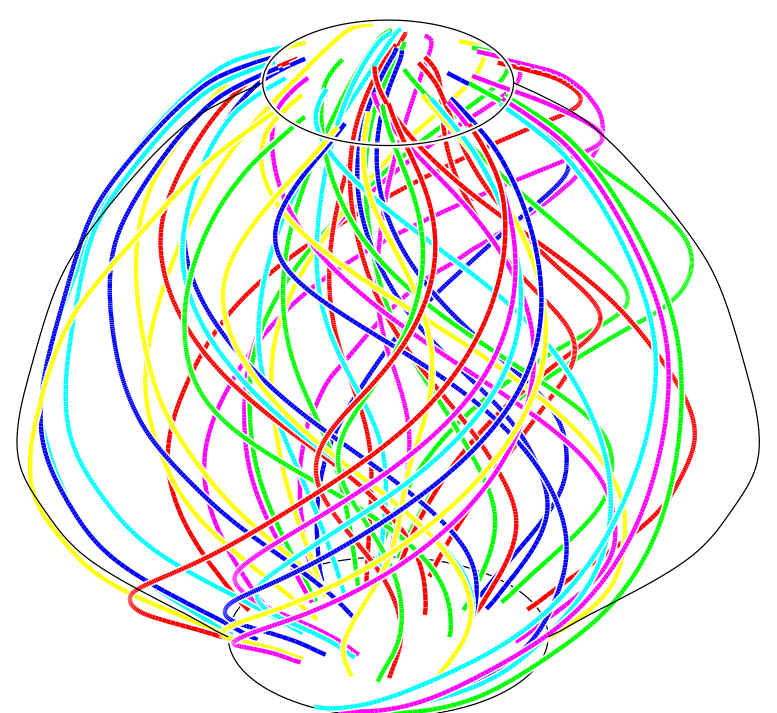

Figure 7. Braided magnetic field in model M1 at time $t=6000 \mathrm{~s}$. The curvature of the loop is neglected (tube axis is vertical in this plot). The circles at top and bottom represent the cross-section of the loop at the TRs $(s=0$ and $s=L)$, and the bottom circle has a radius $R(0)=1 \mathrm{Mm}$. The transverse scale of the plot is expanded by a factor 26.8. In reality the loop length $L_{\text {cor }}=106.3 \mathrm{Mm}$.

(An animation and a color version of this figure are available in the online journal.)

that animations of this type do not directly represent the motion of the plasma, and therefore may be somewhat misleading. The reason is that magnetic reconnection takes place frequently at many sites within the model, so the field lines are not frozen 

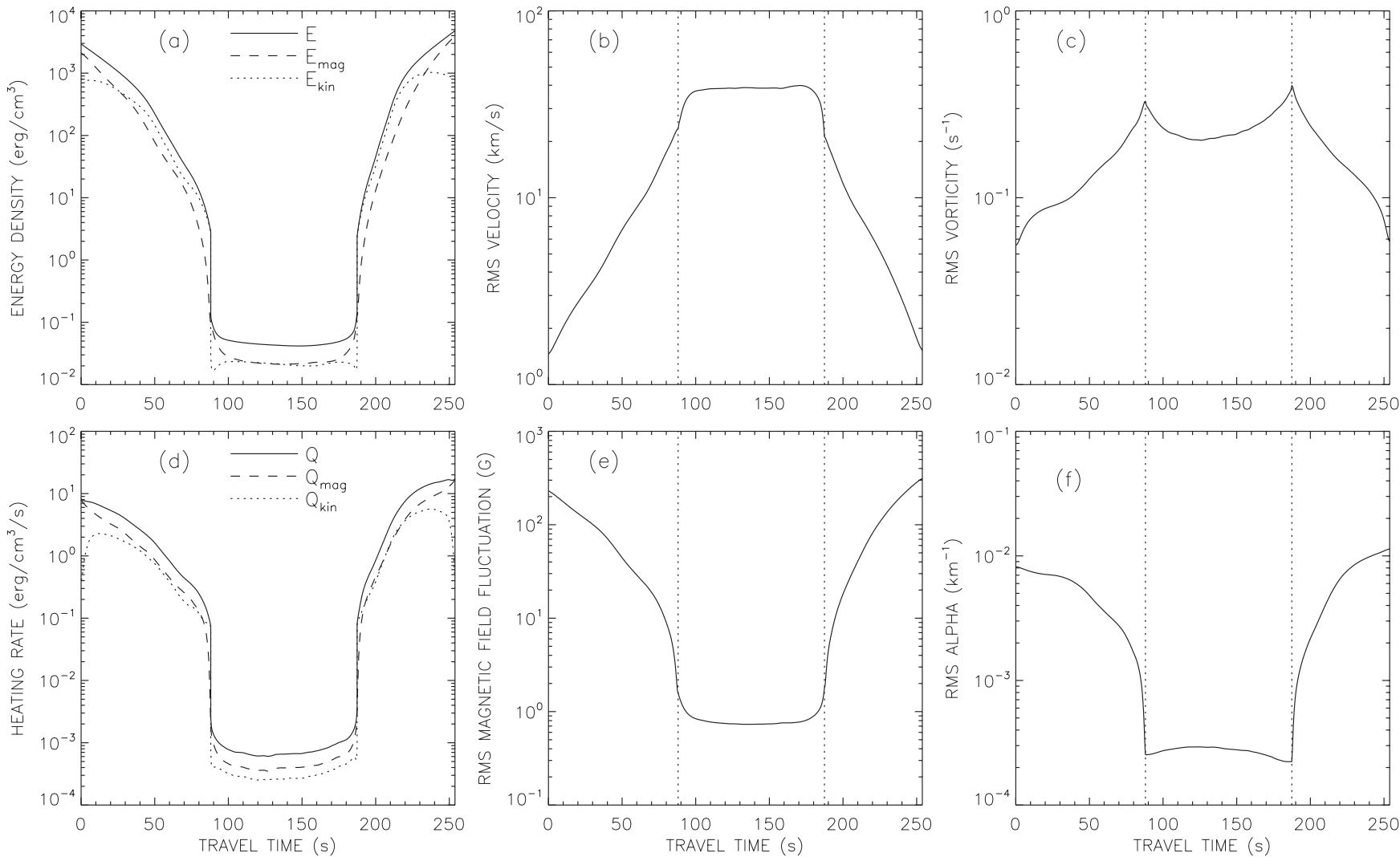

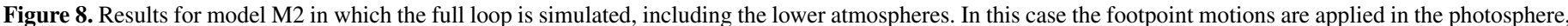

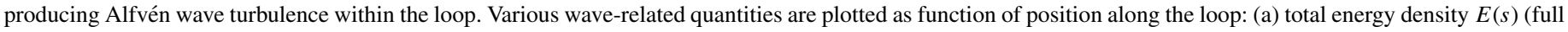

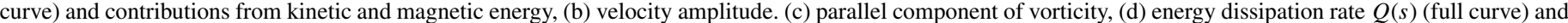

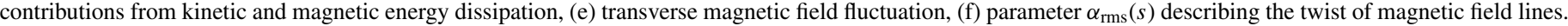

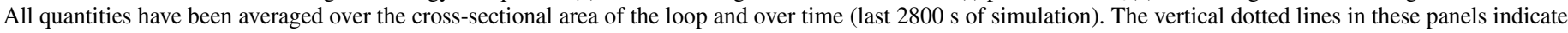
the positions of the TRs.

into the plasma. When tracing field lines from $s=0.44 \mathrm{~L}$ to the ends of the loop, they pass through regions where reconnection is taking place, causing additional displacements. This explains why near the ends of loop the plotted lines are more dynamic than in the middle.

\section{MODELS WITH BOUNDARY CONDITIONS IN THE PHOTOSPHERE}

In this section we consider several MHD models of coronal heating where the lower atmosphere is included in the model (version 2). The magnetic flux tube is assumed to be rooted in small kilogauss flux elements in the photosphere. The two ends of the tube ( $s=0$ and $s=L)$ are now located at the base of the photosphere, $z(0)=z(L)=0$, and the footpoint motions are imposed at this level. The tube radius $R(0)=R(L)=100 \mathrm{~km}$, typical for small-scale magnetic elements in the photosphere. The imposed footpoint motions (see Figure 2(c)) provide a simple model for the interaction of the flux tubes with granulation flow. We consider several models with different values of the imposed photospheric velocity and correlation time. The background atmosphere for these models is shown in Figure 4, and the model parameters are listed in Table 1.

\subsection{Reference Model}

We first describe a reference model M2, which has an imposed footpoint velocity $v_{\mathrm{rms}}=1.48 \mathrm{~km} \mathrm{~s}^{-1}$ and a correlation time $\tau_{c}=60 / \sqrt{2 \pi}=23.9 \mathrm{~s}$. These values are motivated by measurements of the velocities for magnetic bright points, which are proxies for kilogauss flux elements in the photosphere. Chitta et al. (2012) analyzed wideband $\mathrm{H} \alpha$ observations from the Swedish Solar Observatory, and found a velocity amplitude $v_{\text {rms }} \approx 1.3 \mathrm{~km} \mathrm{~s}^{-1}$ and correlation time of about 22-30 s (also see Abramenko et al. 2011). These measurements refer to the motions of the flux tubes as a whole, not their interior motions. Nevertheless, it is reasonable to assume that similar motions occur within the flux tubes. For model M2 the displacement of a fluid parcel in one correlation time is given by $\tau_{c} v_{\mathrm{rms}}=$ $35.4 \mathrm{~km}$, somewhat less than the tube radius of $100 \mathrm{~km}$. The imposed flows cause random intermixing of field lines inside the flux tube, and launch Alfvén waves that travel upward along the tube. The waves reflect due to the gradients in Alfvén speed in the chromosphere and TR, generate turbulence via nonlinear wave-wave interactions, and dissipate their energy in the chromosphere and the corona. The waves are simulated for a period of $3000 \mathrm{~s}$, much longer than the Alfvén wave travel time along the tube (about $250 \mathrm{~s}$ ).

Figure 8 shows various wave-related quantities for model M2. The middle and right panels show the rms values of velocity, vorticity, magnetic field fluctuation, and twist parameter. All these quantities are averaged over the loop cross-section, and over time (last $2800 \mathrm{~s}$ of simulation). Detailed analysis of the simulation results indicates that the twist parameter $\alpha(x, y, s, t)$ fluctuates strongly in space and time. As shown in Figure 8(f), $\alpha_{\text {rms }}(s)$ has its peak in the lower atmosphere. This is due to the strong reflection of Alfvén waves in the chromosphere and TR, which produces counter-propagating waves in the lower 


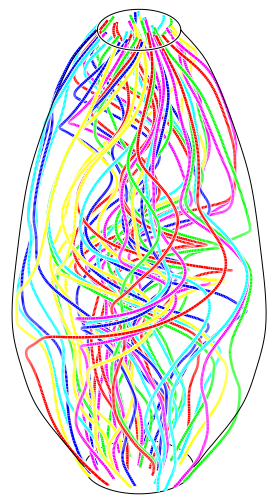

(a)

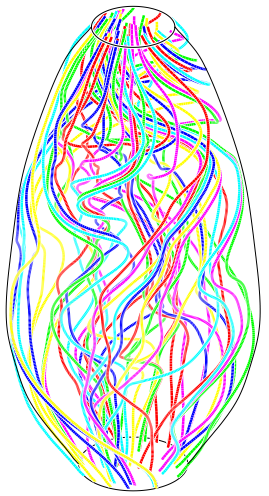

(b)

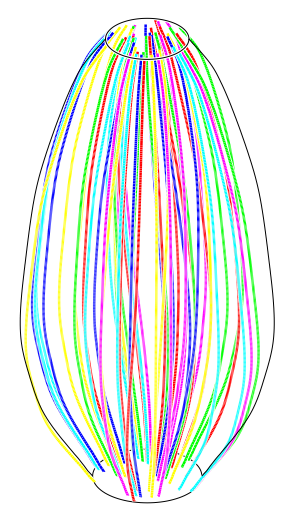

(c)
Figure 9. Coronal magnetic fields in models that include the lower atmosphere. The left and middle panels show results for model M2 at two times in the simulation: (a) $t=2738.5 \mathrm{~s}$, (b) $t=2768.9 \mathrm{~s}$. The transverse scale of the plot is expanded by a factor 26.8. The two circles at top and bottom represent the TRs. These panels demonstrate the rapid variability of braided field over a period of $30 \mathrm{~s}$. Panel (c) shows magnetic field lines for model M4 where virtually no braiding occurs.

(An animation and a color version of this figure are available in the online journal.)

atmosphere and delays the injection of waves into the corona. The non-constancy of $\alpha_{\mathrm{rms}}(s)$ means that the system is far from the force-free equilibrium state. Therefore, in model M2 the coronal field responds dynamically to the footpoint motions.

The model predicts that the coronal magnetic field is braided (also see paper I). Figures 9(a) and (b) show selected field lines in the coronal part of model M2 for two times in the simulation about $30 \mathrm{~s}$ apart. Note that the magnetic field has changed significantly over this period. Therefore, the braided field is highly dynamic, and it is more appropriate to describe such fields as a collection of counter-propagating Alfvén waves.

An animation of Figure 9(a), covering a period of $162 \mathrm{~s}$, is available in the online journal. As mentioned earlier, such animations do not directly represent the motion of the plasma, as reconnection takes place within the volume. Nevertheless, the animation shows how the waves propagate into the coronal loop from both ends and dissipate their energy in the corona.

We computed the time-averaged heating rate $Q(s)$ from the simulation data, and fitted Equation (7) to the results. We then used the one-dimensional loop model to compute the coronal temperature $T(s)$ and pressure $p(s)$. The predicted values of coronal base pressure and temperature are listed in Table 1. Note that unlike for model M1, the model M2 gives realistic values for coronal temperature and pressure. In this paper, we do not further iterate the background atmosphere as we did in paper II.

\subsection{Models with Long Correlation Time}

We now attempt to produce a more quasi-static response to the footpoint motions using version 2 of the code. First we consider a model (M3) in which the correlation time of the footpoint motions is drastically increased, while keeping the velocity amplitude unchanged: $\tau_{c}=1000 / \sqrt{2 \pi}=398.9 \mathrm{~s}, v_{\mathrm{rms}}=$ $1.48 \mathrm{~km} \mathrm{~s}^{-1}$ (see Table 1). In this case the typical displacements of fluid parcels over one correlation time $\tau_{c} v_{\mathrm{rms}}=590 \mathrm{~km}$, significantly larger than the tube radius of $100 \mathrm{~km}$. Therefore, the fluid parcels rotate around in the flow several times before the velocity pattern changes. This type of footpoint motion may not be very realistic, but it is interesting to see how the long correlation time affects the coronal heating rate. Figure 10 shows different quantities plotted as function of position along the loop for this model. Comparing Figure 10(d) with Figure 8(d), we see that the heating rate $Q$ is reduced by a factor of four compared to model M2. The coronal temperature and pressure are also reduced (see Table 1).

Next we consider a model (M4) in which the velocity of the footpoint motions is drastically reduced $\left(v_{\mathrm{rms}}=0.1 \mathrm{~km} \mathrm{~s}^{-1}\right)$ and the correlation time is large $\left(\tau_{c}=398.9 \mathrm{~s}\right)$. In this case the fluid displacement over one correlation time is again smaller than the tube radius $\left(\tau_{c} v_{\text {rms }}=39.9 \mathrm{~km}\right)$, similar to the situation in model M2. In model M4 the waves are simulated for a period of $6000 \mathrm{~s}$. Figure 9(c) shows the magnetic field lines for M4; note that field lines are close to the undisturbed state. Figure 11 shows waverelated quantities plotted as function position along the loop for model M4. Note that the velocity amplitude of the waves is now drastically reduced compared to the reference model M2 (compare Figures 11(b) and 8(b)). Figure 11(f) shows that the average twist parameter $\alpha_{\mathrm{rms}}(s)$ is nearly constant along the flux tube, indicating that the magnetic field is nearly forcefree. Therefore, in model M4 the magnetic field responds quasistatically to the footpoints motions. However, the dissipation rate is only about $10^{-6} \mathrm{erg} \mathrm{cm}^{-3} \mathrm{~s}^{-1}$ (see Figure 11(d)), which is much too low to heat the corona to million-degree temperature. Therefore, in models that include the lower atmosphere it is not possible to simultaneously heat the corona to realistic temperatures and have a quasi-static response to the footpoint motions.

\subsection{Switching Off the Footpoint Motions}

We now consider a model M5 in which the footpoint motions are switched off after $1000 \mathrm{~s}$. Figures 12(a) and (b) show the vorticity of the footpoint motions at the left and right footpoints. The solid and dashed curves correspond to the two driver modes describing the pattern of footpoint motions (see paper I for details). Figures 12(c) and (d) show the Alfvén wave energy density and dissipation rate (averaged over corona) as functions of time in the simulation. Note that the wave energy decreases gradually after $1000 \mathrm{~s}$. We find that the velocity amplitude decreases only gradually with time, and is still about $10 \mathrm{~km} \mathrm{~s}^{-1}$ at time $t=4000 \mathrm{~s}$. If the corona responded quasi-statically to the footpoint motions, all motions within the tube should cease shortly after we freeze the footpoints, however, we find that this is not the case. This illustrates that the braided magnetic field inside the flux tube does not quickly reach a force-free equilibrium state.

The reason that the waves persist for so long is twofold. First, our model includes the lower atmospheres at the two ends of the loop, where the density is much higher than in the corona. Although the corona has a much larger volume, most of the inertia of the waves resides in the lower atmosphere, and so does most of the Alfvén wave energy. Furthermore, in Version 2 the footpoint motions are imposed at the base of the photosphere $\left(\tau_{5000}=1\right)$, which implies that downward propagating waves in the photosphere are perfectly reflected at the ends of the modeled tube. Therefore, when the footpoints are fixed, the waves are trapped inside the flux tube and wave energy is only gradually released into the corona.

The above experiment is somewhat artificial, and only serves to estimate the relaxation time for our model, which has footpoint motions prescribed in the photosphere. In reality the downward propagating waves may travel into the subphotospheric layers, while other waves travel upward from those 

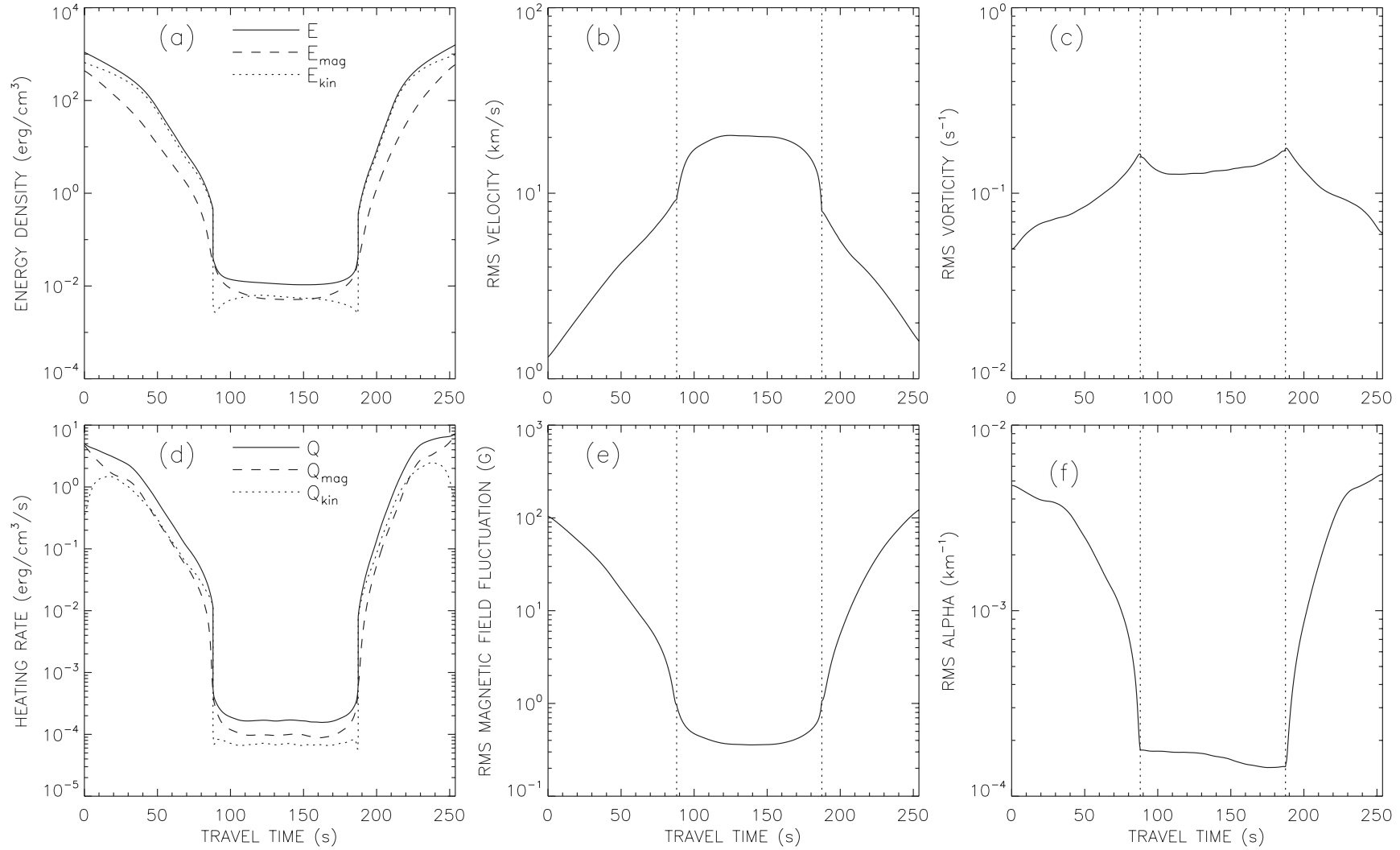

Figure 10. Results for model M3 with standard footpoint velocity $\left(v_{\mathrm{rms}}=1.48 \mathrm{~km} \mathrm{~s}^{-1}\right)$ but long correlation time of the footpoint motions $\left(\tau_{c}=398.9 \mathrm{~s}\right)$. Various wave-related quantities are plotted as function of position along the flux tube: (a) energy densities, (b) velocity amplitude, (c) vorticity, (d) energy dissipation rates, (e) transverse magnetic field fluctuation, (f) twist parameter $\alpha_{\text {rms }}(s)$. The vertical dashed lines indicate the TRs. Note that the wave dissipation $Q(s)$ has been reduced by a factor of $\sim 4$ relative to model M2 (compare with Figure 8(d)).
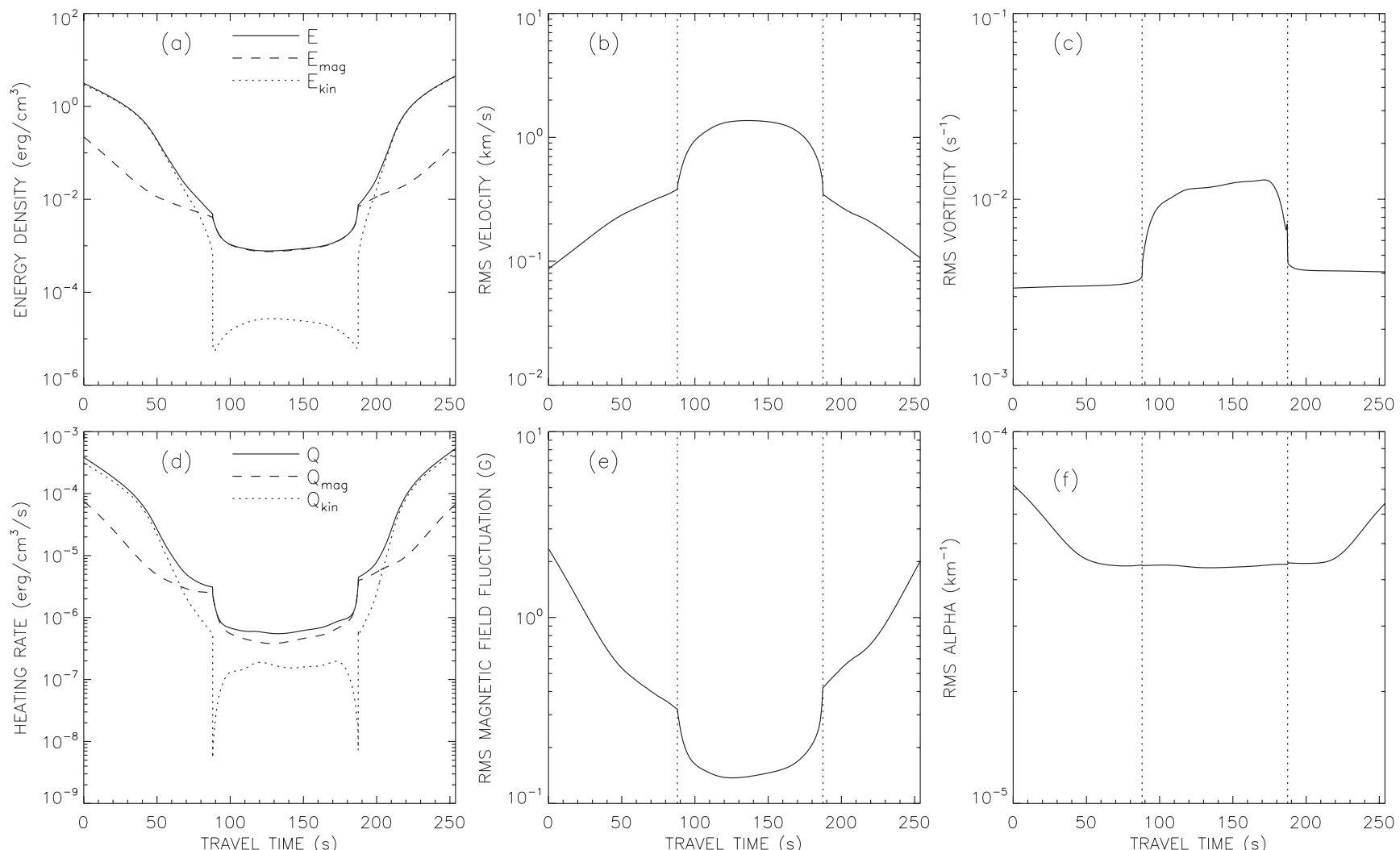

Figure 11. Results for model M4 with greatly reduced footpoint velocity $\left(v_{\mathrm{rms}}=0.1 \mathrm{~km} \mathrm{~s}^{-1}\right)$ and long correlation time $\left(\tau_{c}=398.9 \mathrm{~s}\right)$. Various quantities are plotted as function of position along the tube: (a) energy densities, (b) velocity amplitude, (c) vorticity, (d) energy dissipation rate $Q(s)$, (e) transverse magnetic field, (f) twist parameter $\alpha_{\mathrm{rms}}(s)$. In this case the magnetic field responds quasi-statically to the footpoint motion $\left(\alpha_{\mathrm{rms}}\right.$ is nearly constant), but the heating rate is too low. 

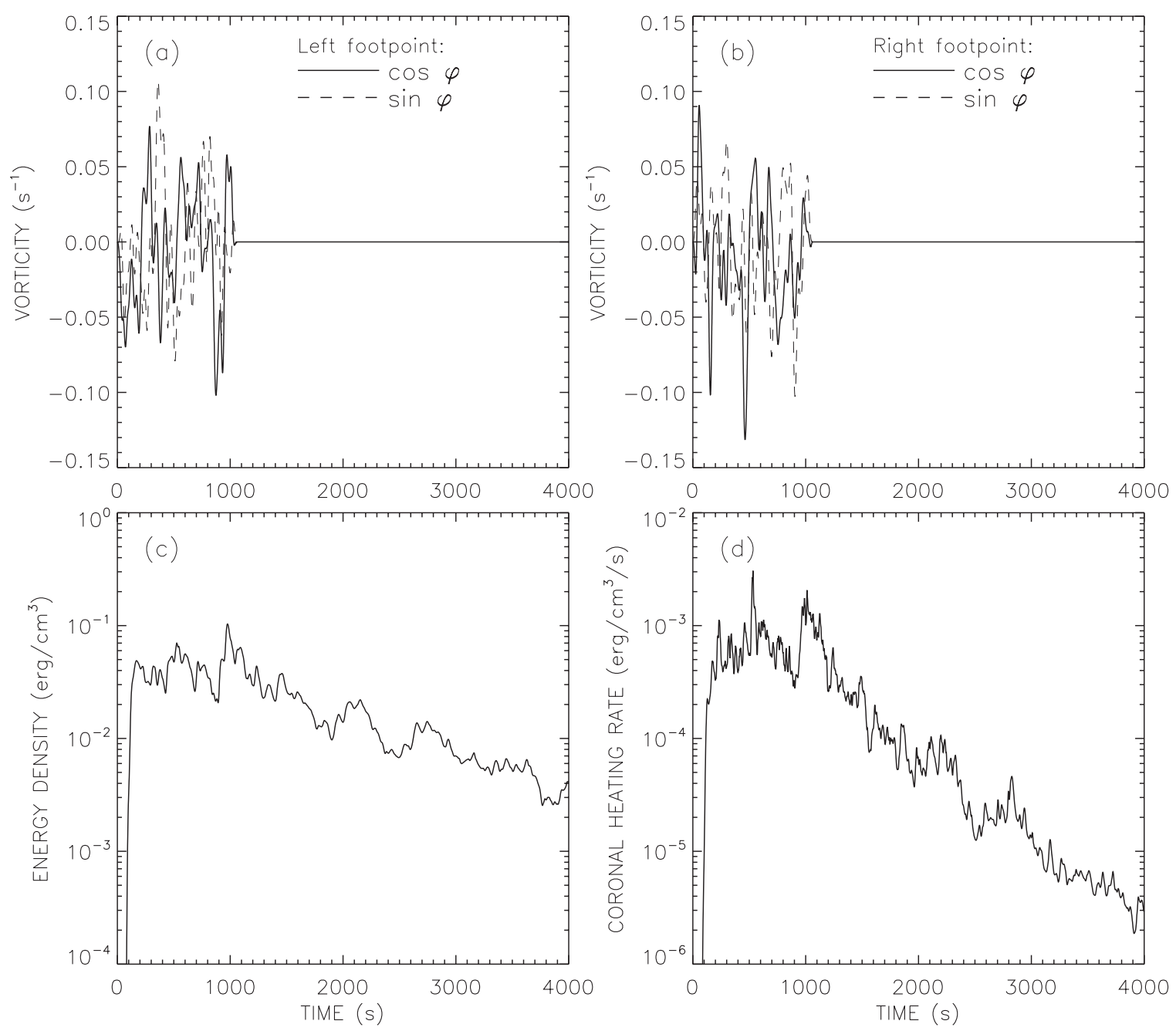

Figure 12. Results for model M5 in which the photospheric driver is switched off after $1000 \mathrm{~s}$. Various quantities are plotted as function of time $t$. The top panels show the vorticities $\omega_{k}(t)$ of the two driver modes that make up the velocity pattern shown in Figure 2 (full and dashed curves are for modes depending on $\cos \varphi$ and $\sin \varphi$, respectively, where $\varphi$ is the azimuth angle). Panel (a) shows the vorticity for the left footpoint $(s=0)$, panel (b) for the right footpoint $(s=L)$. The bottom panels show the coronal wave energy density $E_{\text {cor }}(t)$ and dissipation rate $Q_{\text {cor }}(t)$, which are averages over the coronal volume.

layers. In future modeling the flux tube should be extended deeper into the solar convection zone.

\section{DISCUSSION AND CONCLUSIONS}

Previous modeling of braided magnetic fields has led to the commonly held view that the corona has a tendency to respond quasi-statically to footpoint motions, and that there is more than enough energy available to heat the corona. Our paper challenges this view in two ways. First, we show that due to the inertia of the lower atmosphere the corona tends to respond dynamically to the footpoint motions, so magnetic perturbations are not quasi-static. Second, in models (such as M1) where the effects of the lower atmosphere have been removed, the corona evolves quasi-statically, but there is an energy problem in the sense that such models do not produce sufficient heating (see Section 3). Here we use the important observational constraint that the photospheric footpoint motions have velocities of only $1-2 \mathrm{~km} \mathrm{~s}^{-1}$.

In Section 3 we find that it is possible to obtain a quasi-static response to the footpoint motions (DC heating), provided the lower atmospheres at the two ends of the loop are not included in the model and the "footpoint" motions are applied at the level of the TR. However, the coronal heating rates obtained this way are rather low, $Q \sim 10^{-5} \mathrm{erg} \mathrm{cm}^{-3} \mathrm{~s}^{-1}$. This corresponds to an energy flux into the corona $F_{H} \approx 2 \times 10^{5} \mathrm{erg} \mathrm{cm}^{-2} \mathrm{~s}^{-1}$ at each TR, similar to the value obtained by Bingert \& Peter (2011). The energy flux is much less than the $10^{7} \mathrm{erg} \mathrm{cm}^{-2} \mathrm{~s}^{-1}$ needed to balance the radiative and conductive losses in a typical active region (Withbroe \& Noyes 1977). The coronal temperature predicted for model M1 is only $0.73 \mathrm{MK}$, well below the observed temperatures (3-5 MK). Therefore, the DC heating mechanism described in Section 3 does not provide enough energy to heat the observed active region loop.

The main reason for the low energy flux in model M1 is that there is a rapid "cascade" of magnetic free energy to smaller spatial scales, so that the free energy is dissipated on a timescale of only a few minutes. This cascade is due to the continual fragmentation of the braided field by the imposed "footpoint" motions. Such rapid fragmentation seems consistent with observations of splitting and merging of photospheric flux elements on a timescale of a few minutes (Berger \& Title 1996). Unlike in Parker's (1983) model, the free energy of the braided field is unable to build up over many hours as required to produce the correct heating rate (see discussion in Section 1). Therefore, for a DC heating model to provide enough energy, a way must be found to prevent or delay the cascade of free energy to smaller 
scales. One possibility for enhancing the DC heating rate is that the fragmentation of the photospheric flux elements leads to qualitatively different braiding structure than that generated by the spatially continuous motions at the TR considered here. Motions of discrete flux elements can in principle lead to a coherent braiding, i.e., all the field lines in one element braid in the same way about all the field lines in another element. However, to acquire a significant amount of braiding the flux elements must last a significant amount of time before they themselves fragment and reform. Current sheets will form at the boundaries between flux tubes emanating from different photospheric flux elements (Figure 1). If reconnection at these current sheets occurs rapidly then the braid structure will be lost quickly, reducing the net heating rate. If, however, the sheets burn slowly until secondary instabilities (Dahlburg et al. 2005) are triggered at a high level of braiding, then the expected heating rate will be larger.

Recently, Cirtain et al. (2013) presented data from the Highresolution Coronal Imager (Hi-C), which was launched on a sounding rocket and observed the corona in the $193 \AA$ passband with a spatial resolution of about 0.2 arsec. They claimed to see highly braided magnetic fields at several locations in the observed active region. In our opinion other explanations cannot be ruled out; in particular, for the loops shown in Figure 3 of their paper the observed "braids" may be due to "moss" regions associated with a narrow band of positive polarity flux. However, if we accept Cirtain et al.'s interpretation in terms of highly braided fields, the question arises what sort of footpoint motions are involved in the creation of such fields. For multiple braids to develop, the photospheric flux tubes must retain their identity for many hours as the flux tubes are wrapped around each other (Parker 1983). Again, such long lifetimes of the photospheric flux tubes seem inconsistent with the observations by Berger \& Title (1996), but cannot be ruled out.

In Section 4 we considered models that explicitly include the lower atmospheres at the two ends of the coronal loop. We found that for realistic footpoint velocities $\left(v_{\text {rms }} \approx 1.5 \mathrm{~km} \mathrm{~s}^{-1}\right)$, the footpoint motions produce AWT with heating rates that are sufficient to maintain the corona at temperature of 2-3 MK (also see papers I, II, and III). This model M2 has a dynamic response to the footpoint motions, i.e., AC heating. We conclude that, in order to inject enough energy into the corona, it is necessary to include the lower atmosphere into the model and take into account the dynamic response of the corona to the footpoint motions.

We also presented additional experiments (Section 4.2) in which the correlation time $\tau_{c}$ of the footpoint motions was increased. When the velocity amplitude is kept the same (model M3), the corona still responds dynamically to the footpoint motion, so the correlation time is not the main factor in this dynamic behavior. This model M3 has somewhat unrealistic footpoints motions with fluid parcels that rotate around many times $\left(\tau_{c} v_{\text {rms }} \gg R_{\text {base }}\right)$. A quasi-static response to the photospheric footpoint motions is obtained only when the velocity amplitude is drastically reduced (model M4). In this case the heating rate is very low, and there is not enough energy to heat the corona to realistic temperatures. This illustrates the difficulty of heating the corona using a quasi-static response to the footpoint motions.

In the reference model M2 the twist parameter $\alpha$ varies along the field lines, and its rms value not at all constant (see Figure 8(f)). This is a direct consequence of including the lower atmosphere into the model. The lower atmosphere is much denser than the corona, which greatly increases the inertia in the system. The response of a magnetic flux tube to the footpoint motions depends not so much on the coronal Alfvén travel time $\left(L_{\text {cor }} / v_{A}\right)$, but rather the wave travel time along the entire loop from one photospheric footpoint to the other. The latter is typically in the range 150-300 s, which is comparable to the timescale of the photospheric footpoint motions. Therefore, footpoint motions with timescales $\tau_{f}$ in the range 60-200 s generally excite wave-like disturbances, not quasi-static perturbations.

The models have relatively few free parameters and are well constrained by observations. The shape and strength of the magnetic flux tube are taken from a 3D magnetic model of an observed active region (NOAA 11067), presented in paper II. The model was constructed by "extrapolation" of an SDO/HMI magnetogram, and is an NLFFF model that takes into account the shapes of observed coronal loop. The field strength $B(s)$ along the flux tube was determined by tracing a field line through the NLFFF model and modifying $B(s)$ near the ends to produce kilogauss fields in the photosphere. The photospheric footpoint velocities are assumed to be $1-2 \mathrm{~km} \mathrm{~s}^{-1}$, which are constrained by observations of photospheric magnetic bright points. The background plasma density $\rho(s)$ is constrained by the condition of hydrostatic equilibrium.

According to the AWT model, there is strong wave reflection at the TRs, which produces counter-propagating Alfvén waves already in the lower atmospheres. It is well known that counterpropagating Alfvén waves interact nonlinearly and produce turbulence, as has long been suggested for the solar wind (e.g., Matthaeus et al. 1999; Verdini \& Velli 2007; Cranmer et al. 2007). The turbulence is highly anisotropic with perpendicular length scales much smaller than the parallel ones. Due to such turbulence the motions of the field lines at the TR (i.e., base of the corona) are very complex and are only indirectly related to the photospheric footpoint motions. Furthermore, there is strong amplification of the waves due to the stratification of the lower atmosphere. The velocity increases from about $1.5 \mathrm{~km} \mathrm{~s}^{-1}$ in the photosphere to $30 \mathrm{~km} \mathrm{~s}^{-1}$ at the TR. Therefore, in the context of the coronal heating problem, the corona cannot be treated as an isolated system with "footpoint" motions imposed at the TR (as we did in Section 3). The dynamics of waves in the lower atmosphere must be taken into account.

In summary, the AWT model has several advantages over DC heating models. First, the energy dissipation rates predicted for active region loops are sufficient to explain the observed coronal temperatures, while for DC models the heating rates fall short by at least one order of magnitude as discussed above. Second, the AWT model predicts small perturbations in the directions of the coronal magnetic field (misalignment angles $\sim 4$ degrees), consistent with the fact that observed coronal loops do not show clear evidence for magnetic braiding (Schrijver et al. 1999). Third, the AWT models are consistent with the nonthermal velocities derived from observed spectral line widths, $v_{\mathrm{rms}} \sim 30 \mathrm{~km} \mathrm{~s}^{-1}$. The amplification of the velocities from about $1.5 \mathrm{~km} \mathrm{~s}^{-1}$ at the base of the photospheric flux tubes to $30 \mathrm{~km} \mathrm{~s}^{-1}$ in the TR and low corona is readily explained as an effect of wave propagation in a stratified atmosphere. In DC models the transverse velocities at the coronal base are similar to those at the photospheric footpoints, too small to explain the observed line widths. Hence, the line widths would have to be explained entirely by parallel flows, which seems unlikely. Finally, the AWT model provides a natural explanation for the heating of the magnetic chromosphere (see paper I). 
We thank the anonymous referee for his/her comments which helped to improve this paper. We thank James Klimchuk for discussions that motivated this work. We also thank Stephen Cranmer for discussions and comments that helped improve the paper. We are most grateful to Alex Voss from the School of Computer Science at the University of St. Andrews for his support with the computational work, which was funded by the UK's Engineering and Physical Sciences Research Council (EP/I034327/1). This project is supported under contract NNM07AB07C from NASA to the Smithsonian Astrophysical Observatory (SAO) and SP02H1701R from LMSAL to SAO.

\section{REFERENCES}

Abramenko, V. I., Carbone, V., Yurchyshyn, V., et al. 2011, ApJ, 743, 133

Abramenko, V. I., \& Longcope, D. W. 2005, ApJ, 619, 1160

Antolin, P., \& Shibata, K. 2010, ApJ, 712, 494

Antolin, P., Shibata, K., Kudoh, T., Shiota, D., \& Brooks, D. 2008, ApJ, 688,669

Aschwanden, M. J. (ed.) 2004, Physics of the Solar Corona. An Introduction (Chichester, UK: Praxis Publishing Ltd; Berlin: Springer)

Aschwanden, M. J., \& Nightingale, R. W. 2005, ApJ, 633, 499

Asgari-Targhi, M., \& van Ballegooijen, A. A. 2012, ApJ, 746, 81 (paper II)

Asgari-Targhi, M., van Ballegooijen, A. A., Cranmer, S. R., \& DeLuca, E. E. 2013, ApJ, 773, 111 (paper III)

Berger, M. A. 1993, PhRvL, 70, 705

Berger, M. A. 1994, SSRv, 68, 3

Berger, M. A., \& Asgari-Targhi, M. 2009, ApJ, 705, 347

Berger, T. E., \& Title, A. M. 1996, ApJ, 463, 365

Berger, T., \& Title, A. M. 2001, ApJ, 553, 449

Bingert, S., \& Peter, H. 2011, A\&A, 530, A112

Bingert, S., \& Peter, H. 2013, A\&A, 550, A30

Bourdin, Ph.-A., Bingert, S., \& Peter, H. 2013, A\&A, 555, A123

Cargill, P. J., \& Klimchuk, J. A. 1997, ApJ, 478, 799

Chae, J., Schüle, U., \& Lemaire, Ph. 1998, ApJ, 505, 957

Chitta, L. P., van Ballegooijen, A. A., Rouppe van der Voort, L., DeLuca, E. E., \& Kariyappa, R. 2012, ApJ, 752, 48

Cirtain, J. W., Golub, L., Winebarger, A. R., et al. 2013, Natur, 493, 501

Coleman, P. J., Jr. 1968, ApJ, 153, 371

Craig, I. J. D. 2010, SoPh, 266, 293

Cranmer, S. R., van Ballegooijen, A. A., \& Edgar, R. J. 2007, ApJS, 171, 520

Dahlburg, R. B., Klimchuk, J. A., \& Antiochos, S. K. 2005, ApJ, 622, 1191

De Groof, A., \& Goossens, M. 2002, A\&A, 386, 691

Démoulin, P., van Driel-Gesztelyi, L., Mandrini, C., Klimchuk, J. A., \& Harra, L. 2003, ApJ, 586, 592

DeVore, C. R., Sheeley, N. R., Jr., Boris, J. P., Young, T. R., Jr., \& Harvey, K. 1985, SoPh, 102, 41

DeWijn, A. G., Stenflo, J. O., Solanki, S. K., \& Tsuneta, S. 2009, SSRv, 144,275

Galsgaard, K., \& Nordlund, Å. 1996, JGR, 101, 13445

Golub, L., \& Pasachoff, J. M. 2009, The Solar Corona (Cambridge: Cambridge Univ. Press)

Gómez, D., \& Ferro Fontán, C. 1988, SoPh, 116, 33

Goossens, M., Erdélyi, R., \& Ruderman, M. S. 2011, SSRv, 158, 289

Gudiksen, B. V., \& Nordlund, A. 2002, ApJL, 572, L113
Gudiksen, B. V., \& Nordlund, Å. 2005a, ApJ, 618, 1020

Gudiksen, B. V., \& Nordlund, Å. 2005b, ApJ, 618, 1031

Hendrix, D. L., van Hoven, G., Mikic, Z., \& Schnack, D. D. 1996, ApJ, 470, 1192

Heyvaerts, J., \& Priest, E. R. 1983, A\&A, 117, 220

Heyvaerts, J., \& Priest, E. R. 1992, ApJ, 390, 297

Hollweg, J. V. 1986, JGR, 91, 4111

Hollweg, J. V., Jackson, S., \& Galloway, D. 1982, SoPh, 75, 35

Ionson, J. A. 1985, SoPh, 100, 289

Janse, A. M., \& Low, B. C. 2009, ApJ, 690, 1089

Janse, Å. M., Low, B. C., \& Parker, E. N. 2010, PhPl, 17, 092901

Klimchuk, J. A., Patsourakos, S., \& Cargill, P. J. 2008, ApJ, 628, 1351

Kudoh, T., \& Shibata, K. 1999, ApJ, 514, 493

Longcope, D., \& Strauss, H. 1994, SoPh, 149, 63

Low, B. C. 2010, SoPh, 266, 277

Mandrini, C. H., Démoulin, P., \& Klimchuk, J. A. 2000, ApJ, 530, 999

Martínez González, M. J., Bellot Rubio, L. R., Solanki, S. K., et al. 2012, ApJL, 758, L40

Matsumoto, T., \& Shibata, K. 2010, ApJ, 710, 1857

Matthaeus, W. H., Zank, G. P., Oughton, S., Mullan, D. J., \& Dmitruk, P. 1999, ApJL, 523, L93

Mikić, Z., Schnack, D. D., \& van Hoven, G. 1989, ApJ, 338, 1148

Milano, L. J., Gómez, D. O., \& Martens, P. C. H. 1997, ApJ, 490, 442

Moriyasu, S., Kudoh, T., Yokoyama, T., \& Shibata, K. 2004, ApJL, 601, L107

Ng, C. S., \& Bhattacharjee, A. 1998, PhPl, 5, 4028

Parker, E. N. 1972, ApJ, 174, 499

Parker, E. N. 1983, ApJ, 264, 642

Parker, E. N. 1988, ApJ, 330, 474

Patsourakos, S., \& Klimchuk, J. A. 2006, ApJ, 647, 1452

Peter, H., \& Bingert, S. 2012, A\&A, 548, A1

Priest, E. R., Heyvaerts, J. F., \& Title, A. M. 2002, ApJ, 576, 533

Rappazzo, A. F., Velli, M., Einaudi, G., \& Dahlburg, R. B. 2007, ApJL, 657, L47

Rappazzo, A. F., Velli, M., Einaudi, G., \& Dahlburg, R. B. 2008, ApJ, 677, 1348

Reep, J. W., Bradshaw, S. J., \& Klimchuk, J. A. 2013, ApJ, 746, 193

Schrijver, C. J. 2007, ApJL, 662, L119

Schrijver, C. J., \& Aschwanden, M. J. 2002, ApJ, 566, 1147

Schrijver, C. J., Sandman, A. W., Aschwanden, M. J., \& DeRosa, M. L. 2004, ApJ, 615, 512

Schrijver, C. J., Title, A. M., Berger, T. E., et al. 1999, SoPh, 187, 261

Schrijver, C. J., Title, A. M., Harvey, K. L., et al. 1998, Natur, 394, 152

Schrijver, C. J., \& van Ballegooijen, A. A. 2005, ApJ, 630, 552

Schrijver, C. J., \& Zwaan, C. 2000, Solar and Stellar Magnetic Activity (Cambridge: Cambridge Univ. Press)

Solanki, S. K. 1993, SSRv, 63, 1

Strauss, H. R. 1976, PhFl, 19, 134

Stenflo, J. O. 1973, SoPh, 32, 41

Stenflo, J. O. 1989, A\&ARv, 1, 3

Suzuki, T. K., \& Inutsuka, S.-I. 2006, JGR, 111, A6

Uchida, Y., \& Kaburaki, O. 1974, SoPh, 35, 451

van Ballegooijen, A. A. 1986, ApJ, 311, 1001

van Ballegooijen, A. A., Asgari-Targhi, M., Cranmer, S. R., \& DeLuca, E. E. 2011, ApJ, 736, 3 (paper I)

Verdini, A., \& Velli, M. 2007, ApJ, 662, 669

Wentzel, D. G. 1974, SoPh, 39, 129

Wilmot-Smith, A. L., Hornig, G., \& Pontin, D. I. 2009a, ApJ, 696, 1339

Wilmot-Smith, A. L., Hornig, G., \& Pontin, D. I. 2009b, ApJ, 704, 1288

Winebarger, A. R., \& Warren, H. P. 2005, ApJ, 626, 543

Withbroe, G. L., \& Noyes, R. W. 1977, ARA\&A, 15, 363 